\title{
Toxicity Analysis of Polychlorinated Dibenzofurans through Global and Local Electrophilicities
}

\author{
Utpal Sarkar $^{\mathrm{a}}$, Ramakrishnan Parthasarathi ${ }^{\mathrm{b}}$, Venkatesan Subramanian ${ }^{\mathrm{b} *}$ and Pratim \\ Kumar Chattaraj ${ }^{\mathrm{a}^{*}}$ \\ ${ }^{a}$ Department of Chemistry, Indian Institute of Technology \\ Kharagpur 721302, India \\ ${ }^{\mathrm{b}}$ Chemical Laboratory, Central Leather Research Institute \\ Adyar Chennai 600 020, India
}

Received xxx; Preprint published xxx; Accepted xxx ; Published xxx

\begin{abstract}
Internet Electron. J. Mol. Des. 2003, 1, 000-000

Toxicity of polychlorinated dibenzofurans are correlated with global and local electrophilicities calculated through DFT/6-31G(d) method with B3LYP functionals using both Mulliken and Hirshfeld population analysis schemes. An excellent correlation is observed between the experimental binding affinity values of 31 polychlorinated dibenzofurans with AhR receptors and a linear combination of global and local electrophilicity values.
\end{abstract}

Motivation. To verify the importance of global and local electrophilicities in analyzing toxicity within a QSAR parlance.

Method. Calculation $\omega$ and $\omega_{k}^{+}$of 31 polychlorinated dibenzofurans using Becke's three parameter hybrid density functional, B3LYP, with 6-31G(d) basis set and Mulliken and Hirshfeld population analysis schemes.

Results. A linear relation between the experimental binding affinities $\left(p I C_{50}\right)$ of polychlorinated biphenyls with biosystems and a linear combination of global and local electrophilicity values is observed.

Conclusions. The global and local electrophilicities together can explain the toxicities of polychlorinated dibenzofurans. The beautiful correlation between the binding affinity of these toxins with biosystems and a linear combination of global and local electrophilicity values confirms the importance of charge transfer in analyzing the origin of toxicity.

Keywords. DFT, Dibenzofuran, Toxicity,Electrophilicity.

\author{
Abbreviations and notations \\ QSAR, Quantitative Structure Activity DFT, Density Functional Theory \\ Relationship \\ HAH, Halogenated aromatic hydrocarbon \\ PCDF, Polychlorinated Dibenzofuran \\ FF, Fukui Function \\ IP, Ionization Potential \\ EA, Electron Affinity \\ HOMO, Highest Occupied Molecular Orbital \\ LUMO, Lowest Unccupied Molecular Orbital \\ Dedicated to Professor Danail Bonchev on his $65^{\text {th }}$ birthday \\ * Authors for correspondence E-mails: pkc@chem.iitkgp.ernet.in (PKC), subuchem@hotmail.com \\ (VS) ; Tel: 9103222283304 ; Fax: 913222255303.
}




\section{INTRODUCTION}

Halogenated aromatic hydrocarbons (HAH) like polychlorinated biphenyls, dibenzo-pdioxins, dibenzofurans and benzidines exhibit toxic behavior mainly through their interaction with the biological systems essentially via a protein called AhR [1-3]. Several studies on these compounds have been performed [4-10] in order to gain insights into the actual nature of binding between the HAH and AhR. The interaction is mainly through partial charge transfer augmented by $\pi$ - stacking and in most cases the toxins (HAH) behave as electron acceptors which forces us to treat the electrophilicity as the natural reactivity descriptor [7-9] in the associated quantitative structure activity relationships (QSAR). The amount of charge transfer and the direction of charge flow between the toxin and the biosystem also provide [7-9] important insights into the associated toxicity.

Halogenated aromatic hydrocarbons (HAH) like dibenzofuran occur predominantly as industrial byproducts and the genesis of their toxicity is very complicated and is yet to be understood clearly. Because of the experimental difficulties related to the study of these halogenated aromatic hydrocarbons, QSAR would be helpful to correlate the biological and toxicological activities of these HAHs with different reactivity descriptors.

Compounds are generally screened on the computer in order to select structure with the desired properties using correlation between the structure and the activity of these compounds in QSAR. But it is quite difficult to find good correlation between structure and activity though there exist several models in QSAR. In recent years quantum chemical descriptors are used in QSAR studies because the quantum chemical quantities are able to provide accurate quantitative description of the molecular structures and their chemical properties. Atomic charges, molecular orbital energies, frontier orbital densities, atom- atom polarizabilites, molecular polarizabilities, dipole moments etc., have been used as descriptors within a QSAR parlance.

In the present study we have used global and local electrophilicities as descriptors. According to the density functional theory (DFT) the chemical potential $(\mu)$, and chemical hardness $(\eta)$ are defined as,

$$
\chi=-\mu=-\left(\frac{\partial E}{\partial N}\right)_{v(\vec{r})}
$$

and

$$
\eta=\frac{1}{2}\left(\frac{\partial^{2} E}{\partial N^{2}}\right)_{v(\vec{r})}=\frac{1}{2}\left(\frac{\partial \mu}{\partial N}\right)_{v(\vec{r})}
$$

where $\mathrm{E}$ is the total energy of the system, $\mathrm{N}$ is the number of electrons in the system and $v(\vec{r})$ is the external potential.

The vertical ionization potential (IP) of a system is the change of energy when an electron is removed from the system and variation of the energy when an electron is added to the system is known as electron affinity (EA). In both the cases, electron withdrawing and electron addition, the external potential has to be kept fixed. So using finite difference approximation, eqns. (1) and (2) become, 


$$
\begin{gathered}
\mu=-\frac{(I P+E A)}{2} \\
\eta=\frac{I P-E A}{2}
\end{gathered}
$$

However, to obtain the IP and EA and hence $\mu$ and $\eta$, one needs three energy values $\left(\mathrm{E}_{\mathrm{N},} \mathrm{E}_{\mathrm{N}+1}\right.$ and $\left.\mathrm{E}_{\mathrm{N}-1}\right)$. To save the computational time, we have calculated chemical potential and chemical hardness by using Koopmans' theorem as,

$$
\mu=\frac{E_{L U M O}+E_{H O M O}}{2}
$$

and

$$
\eta=\frac{E_{L U M O}-E_{H O M O}}{2}
$$

where $\mathrm{E}_{\mathrm{LUMO}}$ is the lowest unoccupied molecular orbital's energy and $\mathrm{E}_{\text {Hомо }}$ is the highest occupied molecular orbital's energy.

Using $\mu$ and $\eta$ Parr et al have defined a new quantum chemical descriptor, known as electrophilicity index $(\omega)$ which measures the propensity to absorb electrons and is defined as [11]

$$
\omega=\frac{\mu^{2}}{2 \eta}
$$

The Fukui function is defined within a DFT framework as [12],

$$
f(\vec{r})=\left(\frac{\partial \rho(\vec{r})}{\partial N}\right)_{v(\vec{r})}=\left(\frac{\delta \mu}{\delta v(\vec{r})}\right)_{N}
$$

where $\rho(\vec{r})$ is the electron density.

Recently Chattaraj et al provide [13, 14] a unified treatment of chemical reactivity and selectivity through a generalized philicity concept by using a resolution of identity. This local philicity index is given as

$$
\omega^{\alpha}(\vec{r})=\omega f^{\alpha}(\vec{r})
$$

or its condensed- to- atom variants for the atomic site $k$ in a molecule is defined as

$$
\omega_{k}^{\alpha}=\omega f_{k}^{\alpha}
$$

where $\alpha=+,-, 0$ refer to nucleophilic, electrophilic and radical attacks respectively where $f_{k}^{\alpha}$ is the corresponding Fukui function.

In the present work an attempt has been made in correlating the binding affinities of 31 polychlorinated dibenzofurans in terms of their global and local electrophilicity values. 


\section{COMPUTATIONAL DETAILS}

All the structures of the polychlorinated dibenzofurans are optimized to their lowest energy states using the Gaussian 03 software package. During the process of optimization no symmetry restriction has been made use of. The frequency calculation has been followed by the optimization procedure. The presence of zero imaginary frequency in the frequency calculation on the optimized geometries confirms that the structures are minima on the potential energy surface and hence the lowest energy ground states. Becke's three- parameter hybrid density functional, B3LYP, with basis set 6-31G(d) has been used. Fukui functions have been calculated using the direct method proposed by Contreras et al [15] and using the Mulliken population analysis (MPA) scheme as well as with the Hirshfeld population analysis (HPA) scheme [16] using DMOL package [17] with BLYP/DN method.

\section{RESULTS AND DISCUSSION}

Optimized structures with the atom- numbering schemes of all the polychlorinated dibenzofurans (PCDF) studied in the present work are provided in Figure 1 whereas their geometrical parameters are presented in Table 1. In our previous works [7-9] related to the effect of conformational flexibility on toxicity of polychlorinated biphenyls and benzdines, we highlighted the importance of the global electrophilic behavior characterized by $\omega$ as well as that of the most probable site given by $\omega_{k}^{\alpha}$. Table 2 reports the total energy, hardness, chemical potential, global electrophilicity and the local electrophilicity (both MPA and HPA) for the centre with the maximum value. In order to ascertain the actual direction of the charge transfer we have also presented the hardness, chemical potential and global electrophilicity of the selected nucleic acid (NA) bases (adenine, thymine, guanine, cytosine and urasil) and DNA base pairs (GCWC, ATH) in Table 3. Since electrons flow from a system of higher chemical potential (lower electronegativity) to another of lower chemical potential (higher electronegativity) PCDFs will act as electron acceptors and the bases/base pairs as electron donors. An analysis of the joint hardness $\left(\eta_{A B}=\frac{I P_{\min }-E A_{\max }}{2}\right)$ also provides (not shown here) the same inference. Therefore we have to take $\omega_{k}^{+}(\mathrm{k}$ is the site having the maximum $\omega^{+}$value) into consideration. In case the toxin is an electron donor (like benzidines) we should consider $\omega_{k}^{-}$. It is imperative to develop a QSAR based on two related parameters, one global $(\omega)$ and one local ( $\omega_{k}^{+}$for electron acceptors and $\omega_{k}^{-}$for electron donors). Figure 2 shows the excellent correlation between the observed binding affinity (BA) of the toxins with AhR receptors $[4,5]$ in terms of their $\left[\mathrm{pIC}_{50}=-\log \left(\mathrm{IC}_{50}\right)\right]$ values where $\mathrm{IC}_{50}$ is the inhibitor concentration [6] needed to reduce the enzyme activity by $50 \%$ and the corresponding calculated BA values in terms of $\omega$ and $\omega_{k}^{+}$. The correlation is very good in the cases of the local descriptors calculated using HPA ( $\mathrm{R}=0.8742$, fig. $2 \mathrm{a})$ and MPA ( $\mathrm{R}=0.8184$, fig. $2 \mathrm{~b})$ and also by using the BA values normalized [6] to that of 2, 3, 7, 8- tetrachloro dibenzofuran (TCDF) (figs. $2 \mathrm{c}(\mathrm{R}=0.8722, \mathrm{HPA}$ ) and $2 \mathrm{~d}(\mathrm{R}=0.8157$, MPA)). These correlations are better than both one and multi- parameter fits reported in reference 10. Incidentally their [10] finding of reliability of softness as the toxicity descriptor is a manifestation of the maximum hardness principle [18] as was highlighted in the toxicity analysis of polychlorinated biphenyls [7, 8] and benzidine [9] The superiority of the present QSAR not only rests on better regression but also on the transparent understanding of the DFTbased global and local reactivity descriptors used here vis- á- vis the importance of the electron 
transfer ability between a toxin and a biosystem in gaining insights into the overall toxicity. Further work along this line is in progress in our laboratories.

\section{Conclusions}

Since the toxicity of halogenated aromatic hydrocarbons mainly originates from their interaction with biosystems essentially through electron transfer it is expected that global and local electrophilicities would provide important insights into their toxic behavior. An excellent linear correlation between the binding affinity of polychlorinated dibenzofurans with AhR receptors and a linear combination of global and local electrophilicities is observed. The genesis of this beautiful quantitative structure activity relationship is also now well understood.

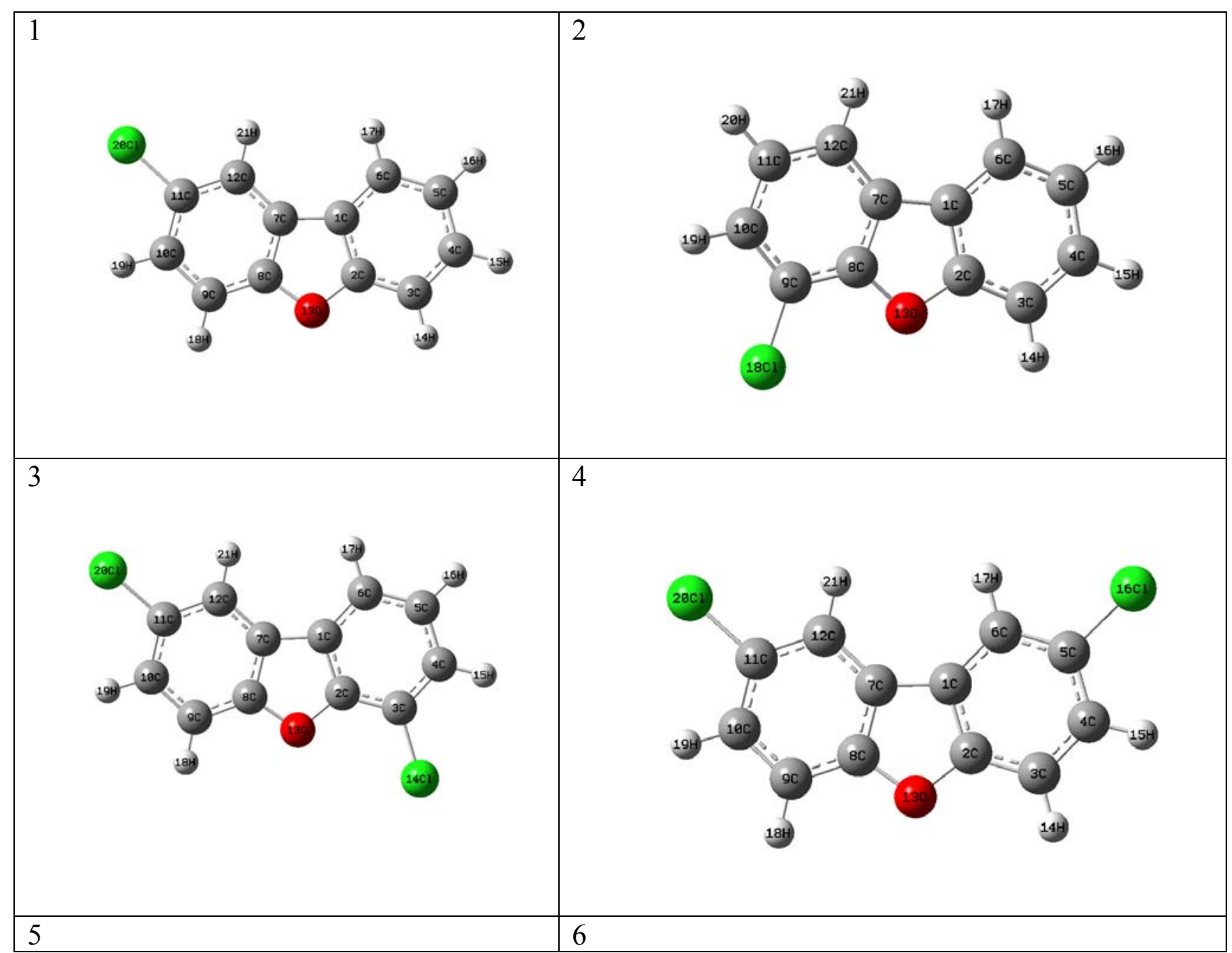




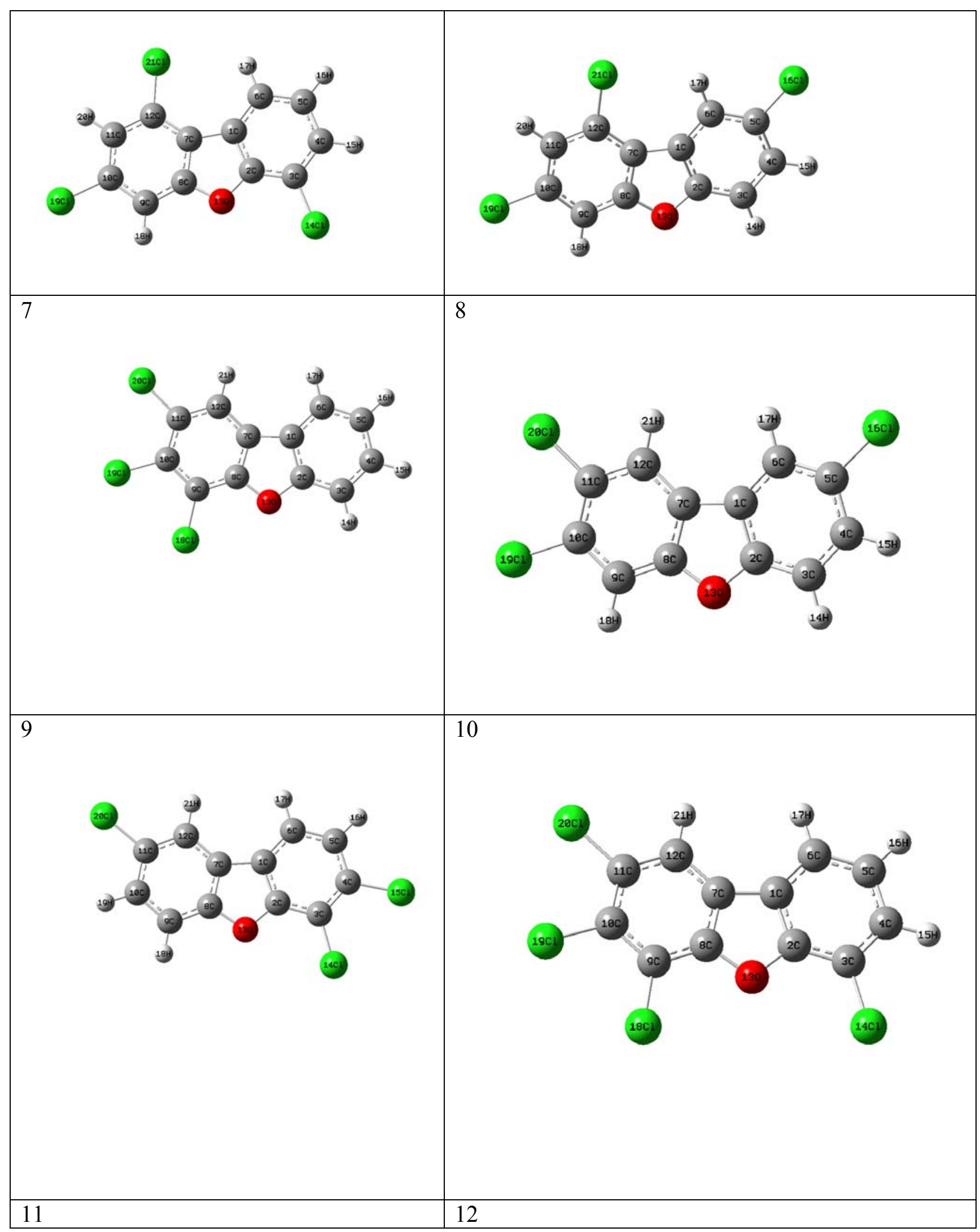




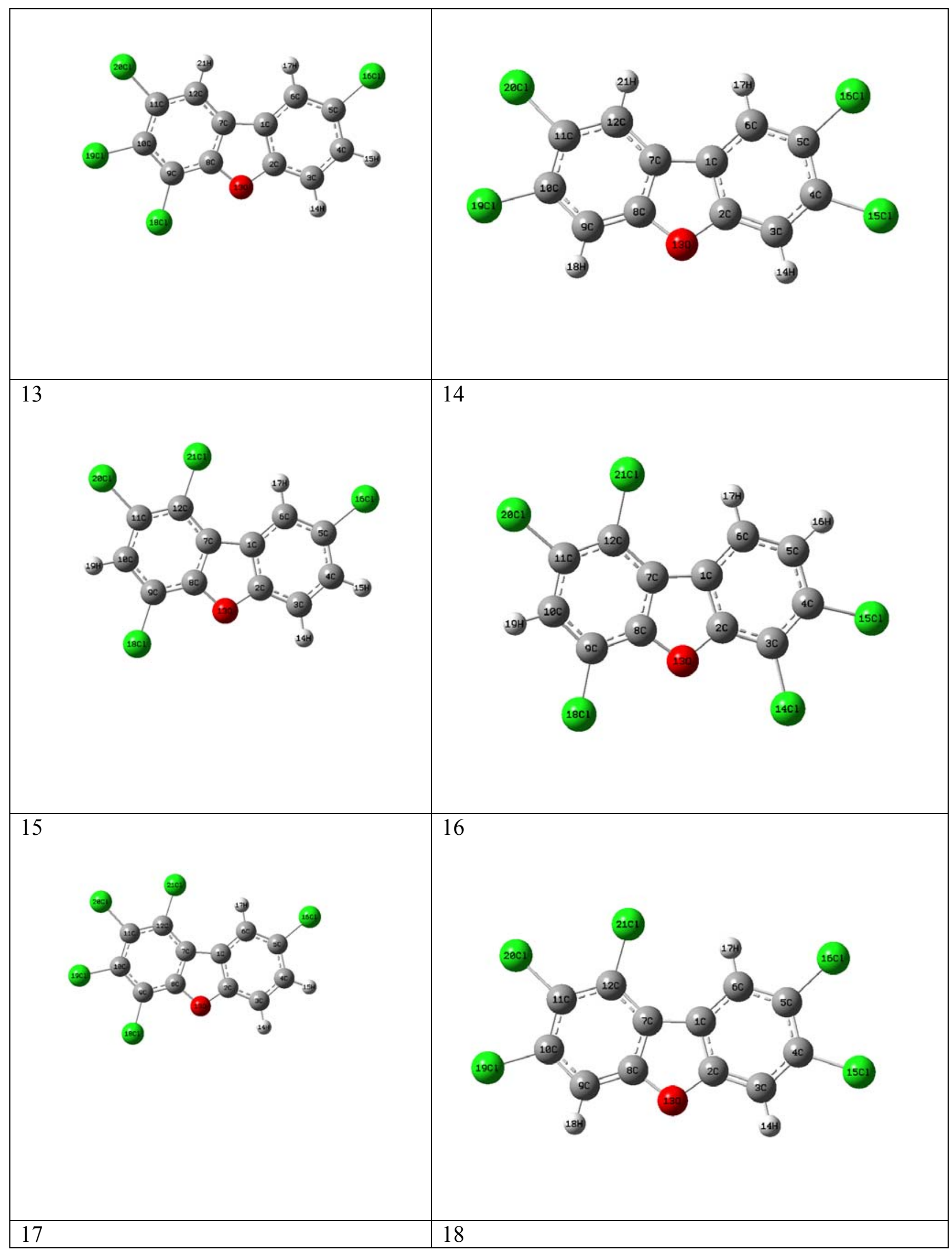

BioChem Press 


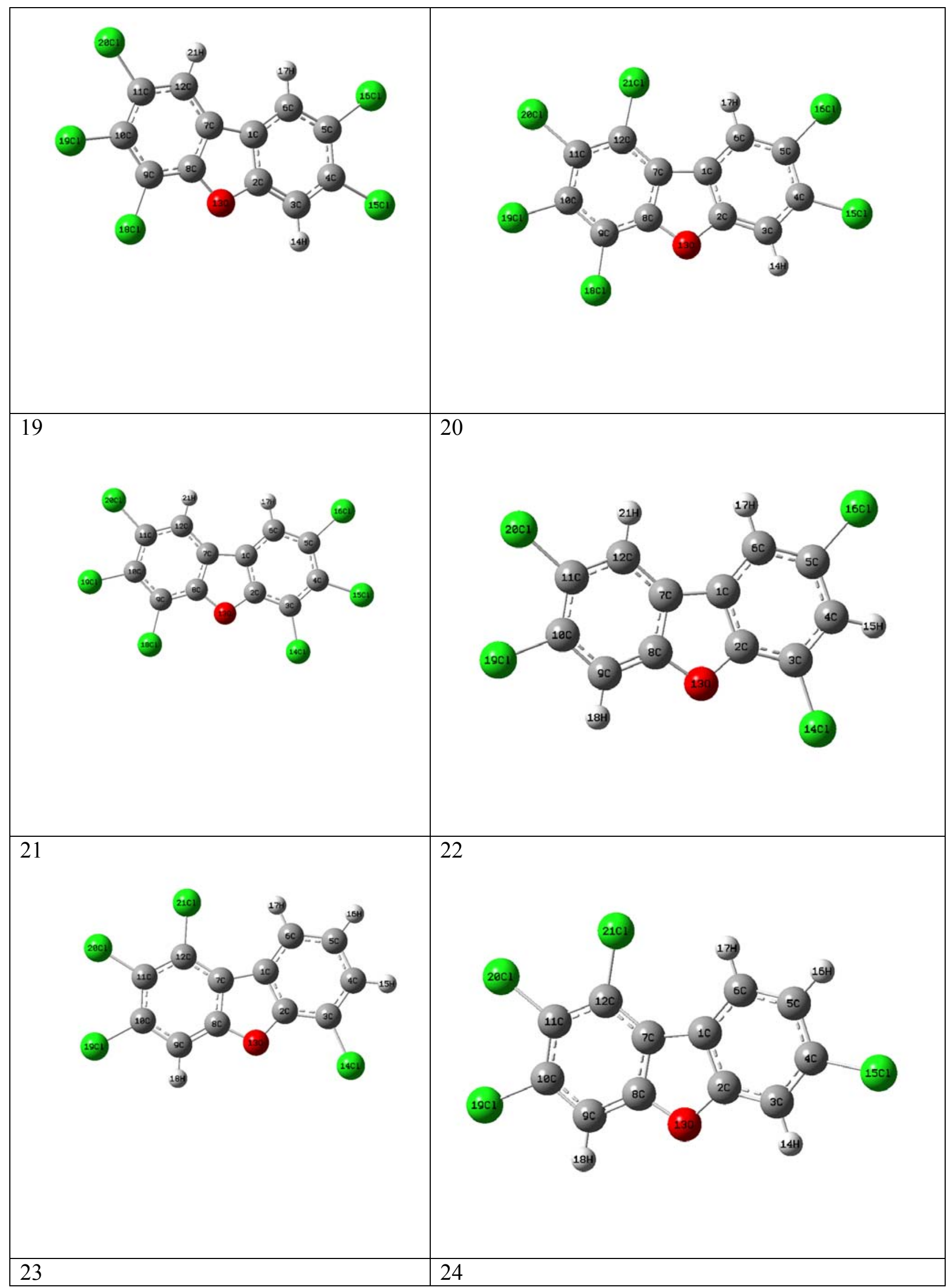




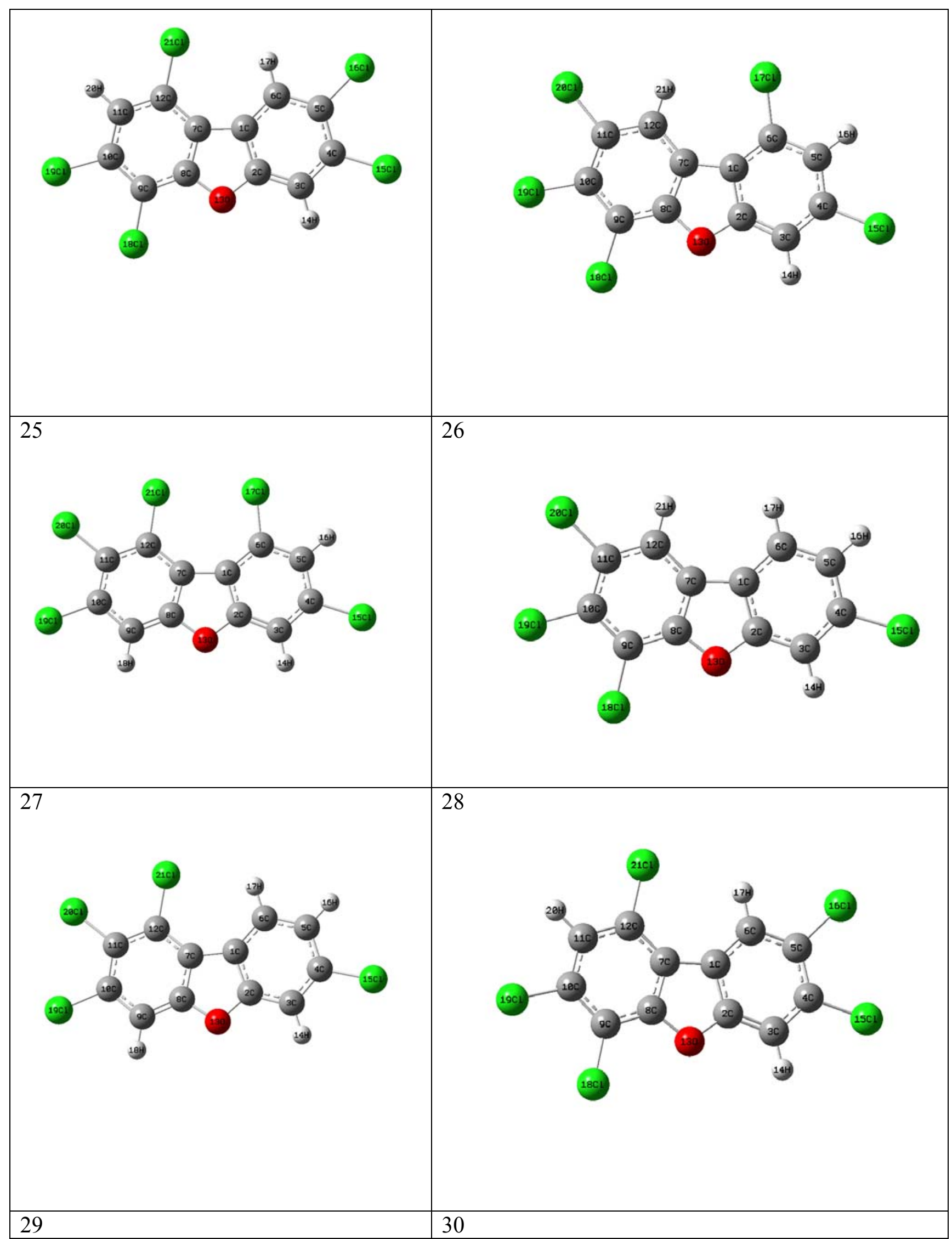




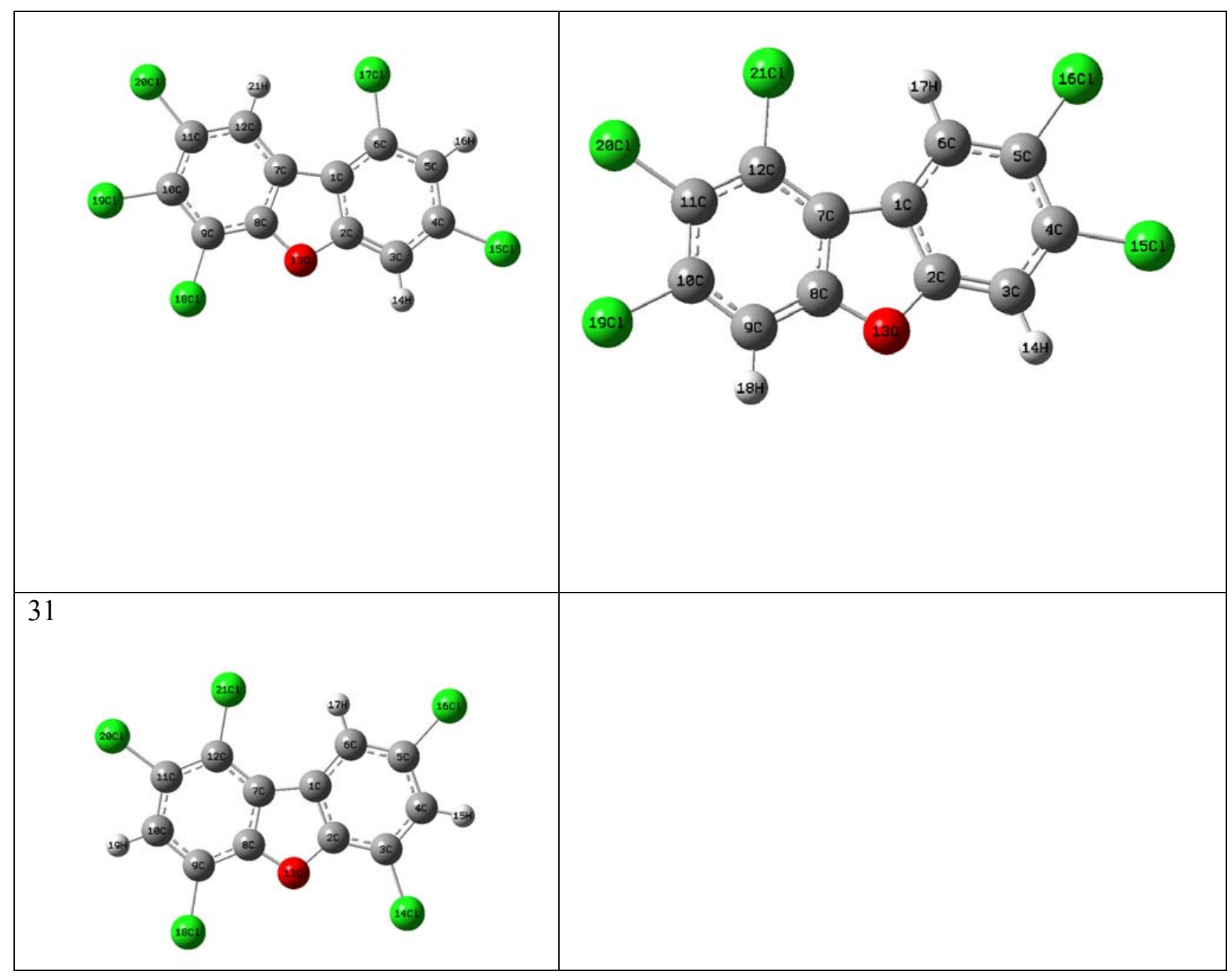

Figure 1. Geometries of different polychlorinated dibenzofurans with atom numbering 


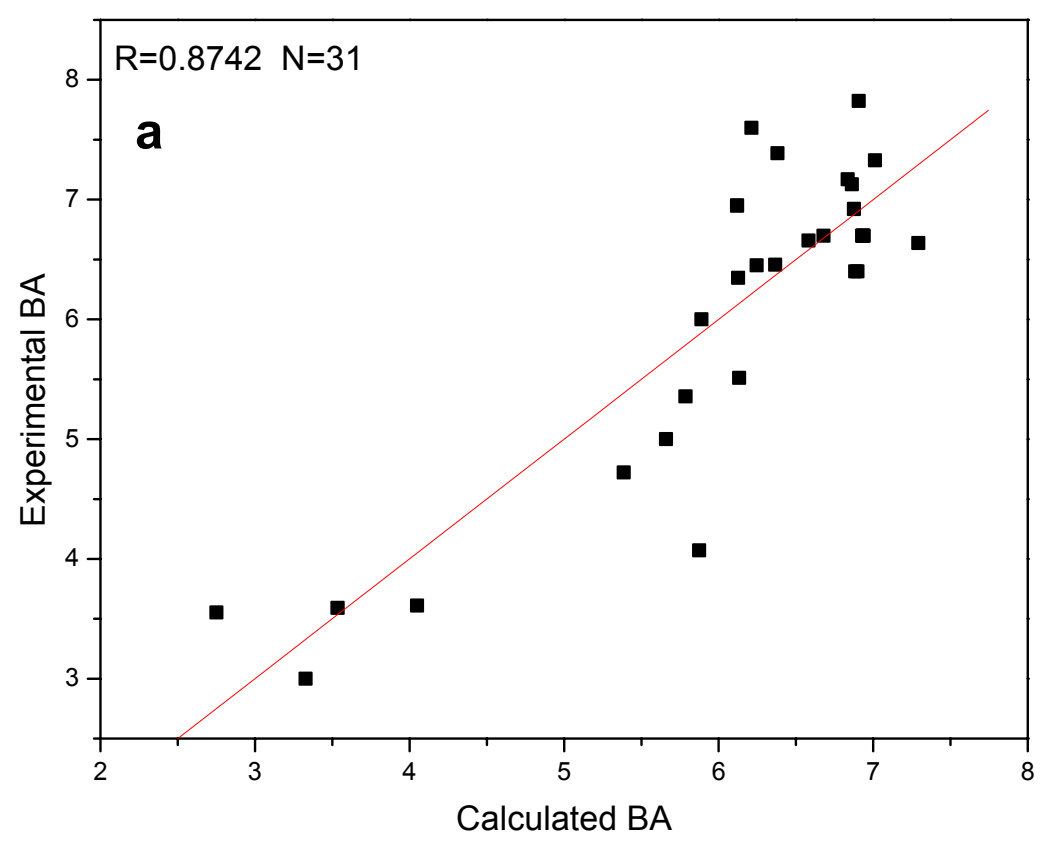

Calculated $\mathrm{BA}=-3.8314+613.4692 * \omega+15.1564 * \omega_{\max }^{+}$

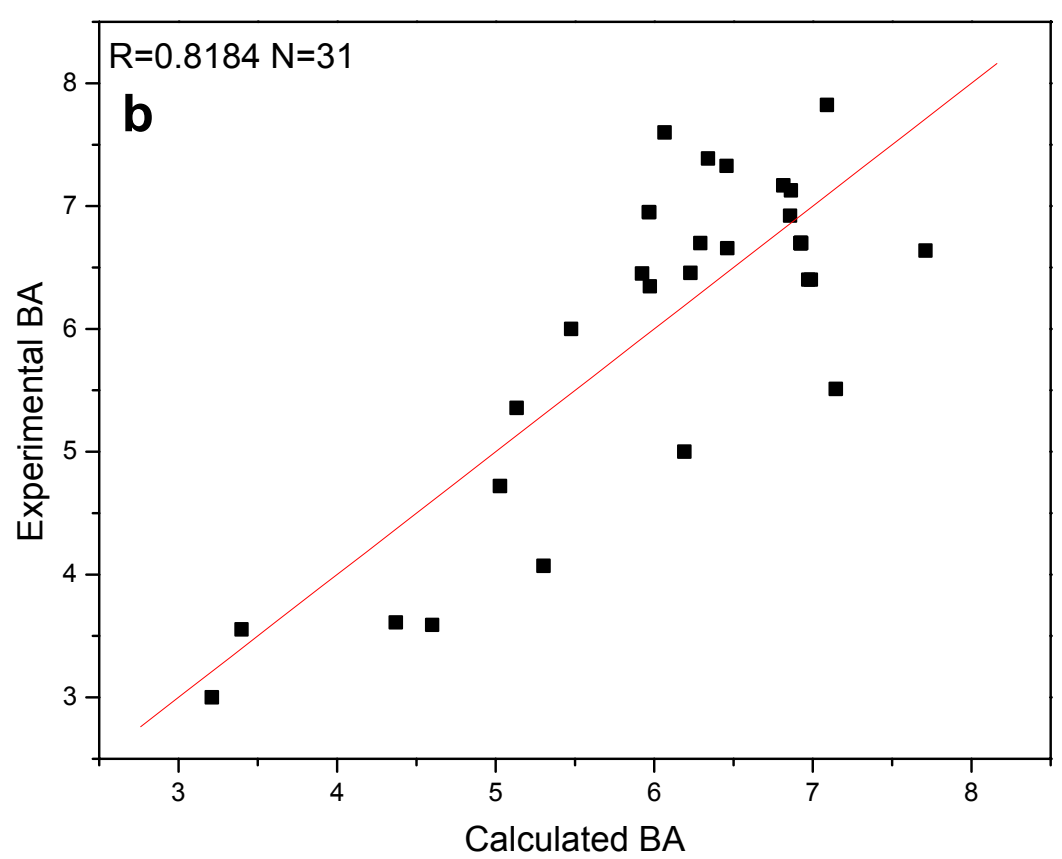

Calculated BA $=-4.5426-3.4852 * \omega+78.7406 * \omega_{\max }^{+}$ 


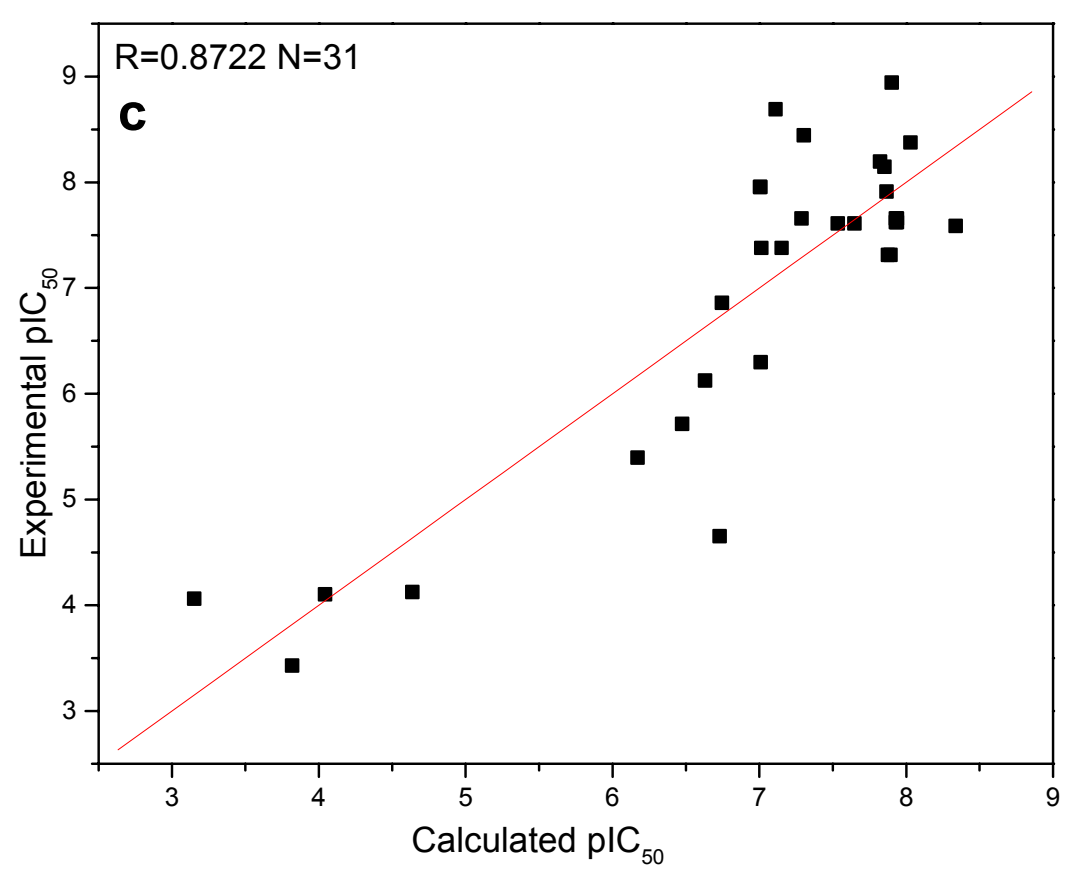

Calculated $\mathrm{pIC}_{50}=-4.3483+16.6328 * \omega+706.8821 * \omega_{\max }^{+}$

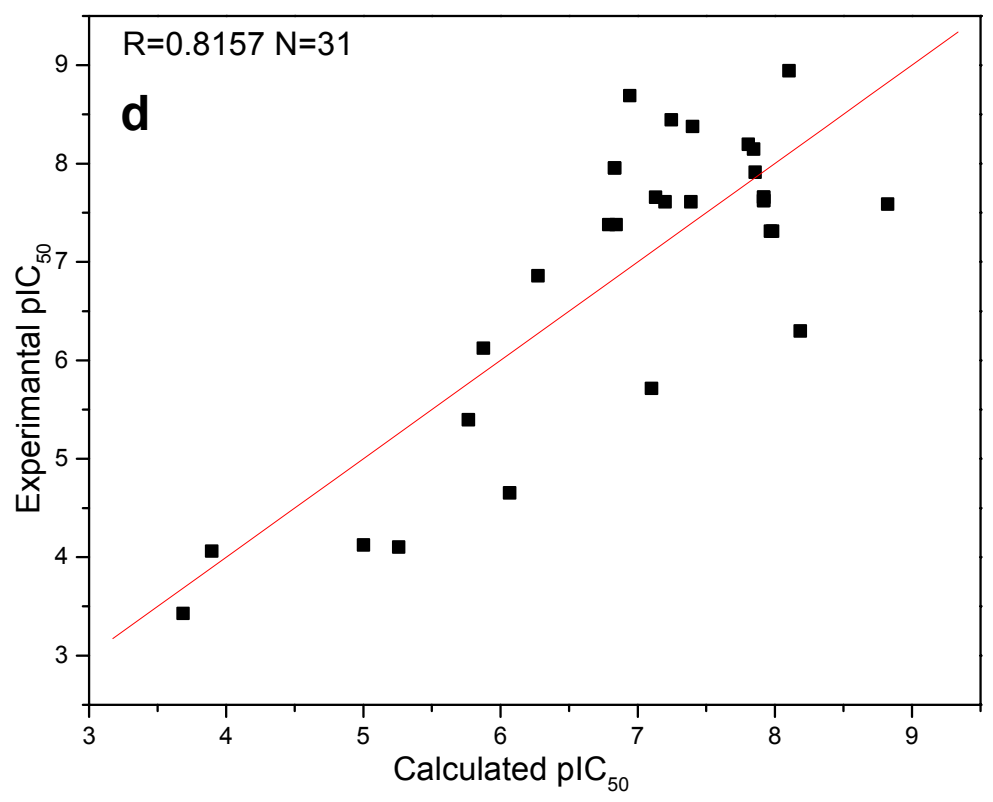

Calculated $\mathrm{pIC}_{50}=-5.1966+88.2057 * \omega+8.5797 * \omega_{\max }^{+}$ 
Figure 2. Correlation between calculated and experimental binding affinities $\left(\mathrm{pIC}_{50}\right)$ : a) HPA, b) MPA; corresponding values normalized to that of (TCDF) c) HPA, d) MPA.

\section{Acknowledgments}

We thank CSIR, New Delhi for financial assistance. PKC thanks Dr. Ovidiu Ivanciuc for kindly inviting him to submit this article in this Special Issue.

\section{References}

[1] S. Safe, S. Bandiera, T. Sawyer, B. Zmudzka, G. Mason, M. Romkes, M. A. Denomme, J. Sparlinf, A. B. Okey and T. Fujita, "Effects of Structure on Binding to 2, 3, 7, 8- TCDD Cytosolic Receptor Protrin and AHH Induction- Halogenated Biphenyls", Environ. Health. Perspect. 1985, 61, 21.

[2] A. Poland and J. C. Knutson, “2, 3, 7, 8- Tetrachlorodibenzo-p-dioxin and Related Halogenated Aromatic Hydrocarbons: Examination of the Mechanism of Toxicity", Annu. Rev. Pharmacol. Toxicol. 1982, 22, 517.

[3] C. Hansch, D. Hoekman, A. Leo, D. Weininger and C. D. Selassie, "Chem- bioinformatics: Comparative QSAR at the Interface Between Chemistry and Biology", Chem. Rev. 2002, 102, 783.

[4] S. Bandiera, T. W. Sawyer, M. A. Campbell, T. Fujita and S. Safe, "Competitive Binding to the Cytosolic 2, 3, 7, 8 TCDD Receptor: Effects of Structure on the Affinities of Substituted Halogenated Biphenyls- a QSAR Analysis”, Biochem. Pharmacol. 1983, 32, 3803.

[5] S. Bandiera, T. W. Sawyer, M. Romkes, B. Zmudzka, L. Safe, G. Mason, B. Keys and S. Safe, "Polychlorinated Dibenzofurans (PCDFs): Effects of Structure on Binding to 2, 3, 7, 8- TCDD Cytosolic Receptor Protrin. AHH Induction and Toxicity", Toxicology, 1984, 32. 131.

[6] C. L. Waller and J. D. McKinney, "Three- Domensional Quantitative Structure- Activity Relationship of Dioxins and Dioxin- like Compounds: Model Validation and Ah Receptor Characterization", Chem. Res. Toxicol., 1995, 8, 847.

[7] R. Parthasarathi, J. Padmanabhan, V. Subramanian, B. Maiti, P. K. Chattaraj, "Chemical Reactivity Profiles of Two Selected Polychlorinated Biphenyls", J. Phys. Chem. A, 2003, 107, 10346.

[8] R. Parthasarathi, J. Padmanabhan, V. Subramanian, B. Maiti, P. K. Chattaraj, "Toxicity Analysis of 33'44'5 - Pentachloro Biphenyl Through Chemical Reactivity and Selectivity Profiles" Curr, Sci., 2004, 86, 535.

[9] R. Parthasarathi,J. Padmanabhan, V. Subramanian, U. Sarkar, B. Maiti, P. K. Chattaraj, "Toxicity Analysis of Benzidine Through Chemical Reactivity and Selectivity Profiles: A DFT Approach", Internet Electron. J. Mol. Desg., 2003, $2,798$.

[10] S. Arulmozhiraja and M. Morita, "Structure- Activity Relationships for the Toxicity of Polychlorinated Dibenzofurans: Approach Through Density Functional Theory- Based Descriptors", Chem. Res. Toxicol., 2004, 17, 348. 
[11] R G. Parr, L. v. Szentpaly and S. Liu, Electrophilicity Index J. Am. Chem. Soc. 1999, 121, 1922.

[12] R.G. Parr and W. Yang, "Density Functional Approach to the Frontier-Electron Theory of Chemical Reactivity" J. Am. Chem. Soc. 1984, 106,4049.

[13] P. K. Chattaraj, B. Maiti and U. Sarkar, "Philicity: A Unified Treatment of Chemical Reactivity and Selectivity", J. Phys. Chem. A, 2003, 107, 4973.

[14] P. K. Chattaraj and U. Sarkar, "A New Generalized Concept of Chemical Reactivity and Selectivity" Internet Electron. J. Mol. Desg. In Press.

[15] R.R. Contreras, P. Fuentealba, M. Galvan, and P. Perez, "A Direct Evaluation of Regional Fukui Functions in Molecules" Chem. Phys. Lett. 1999, 304, 405.

[16] F. L. Hirshfeld, "Bonded-atom fragments for describing molecular charge densities", Theor. Chim. Acta. 1977, 44, 129.

[17] DMOL ${ }^{3}$, Accelrys, Inc., San Diego, California.

[18] R. G. Parr and P. K. Chattaraj, "Principle of Maximum Hardness", J. Am. Chem. Soc.,

Communication, 1991, $113,1854$. 


\section{Biographies}

U. Sarkar received his B.Sc. (Physics Honours) from the University of Burdwan and M.Sc. (Physics) from the Visva-Bharati University, Santiniketan. Then he joined the research group of Professor P. K. Chattaraj for his Ph.D. degree in the Chemistry Department, Indian Institute of Technology, Kharagpur as a CSIR (Government of India) Fellow.

R. Parthasarathi is Senior Research Fellow in Chemical Laboratory, Central Leather Research Institute at Chennai. After obtaining a MSc., Degree in Biochemistry doing Ph.D. in University of Madras under the guidance of Dr. V. Subramanian.

V. Subramanian is a Scientist in Chemical Laboratory, Central Leather Research Institute at Chennai. He received his Ph.D. degree from University of Madras. Dr Subramanian's research interests are $a b$ initio quantum chemistry and molecular modeling and simulation of biomolecules.

P. K. Chattaraj obtained his Ph.D. degree from Indian Institute of Technology, Bombay. Subsequently he did his postdoctoral research in the University of North Carolina at Chapel Hill. He is currently a Professor of chemistry in Indian Institute of Technology, Kharagpur. Professor Chattaraj's research interests include density functional theory, nonlinear dynamics, ab initio calculations and the theory of chemical reactivity. He was a visiting faculty at several universities throughout the globe. Professor Chattaraj is a Fellow of the Indian Academy of Sciences, Bangalore. He is a member of the Editorial Board of Journal of Chemical Sciences.

Table 1: Geometrical Parameters of Different Polychlorinated Dibenzofurans

\begin{tabular}{|c|c|c|c|}
\hline Molecule & Bond Length (A) & Angle (Degree) & Dihedral Angle (Degree) \\
\hline 1 & $\begin{array}{l}\mathrm{C} 2-\mathrm{C} 1=1.408 \\
\mathrm{C} 3-\mathrm{C} 2=1.388 \\
\mathrm{C} 4-\mathrm{C} 3=1.396 \\
\mathrm{C} 5-\mathrm{C} 4=1.405 \\
\mathrm{C} 6-\mathrm{C} 5=1.393 \\
\mathrm{C} 7-\mathrm{C} 1=1.452 \\
\mathrm{C} 8-\mathrm{C} 7=1.408 \\
\mathrm{C} 9-\mathrm{C} 8=1.387 \\
\mathrm{C} 10-\mathrm{C} 9=1.395 \\
\mathrm{C} 11-\mathrm{C} 10=1.403 \\
\mathrm{C} 12-\mathrm{C} 11=1.392 \\
\mathrm{O} 13-\mathrm{C} 8=1.375 \\
\mathrm{H} 14-\mathrm{C} 3=1.085 \\
\mathrm{H} 15-\mathrm{C} 4=1.086 \\
\mathrm{H} 16-\mathrm{C} 5=1.086 \\
\mathrm{H} 17-\mathrm{C} 6=1.086 \\
\mathrm{H} 18-\mathrm{C} 9=1.085 \\
\mathrm{H} 19-\mathrm{C} 10=1.084 \\
\mathrm{Cl} 20-\mathrm{C} 11=1.762 \\
\mathrm{H} 21-\mathrm{C} 12=1.085\end{array}$ & $\begin{array}{l}\text { C3-C2-C1=123.309 } \\
\text { C4-C3-C2=116.627 } \\
\text { C5-C4-C3=121.370 } \\
\text { C6-C5-C4=121.069 } \\
\text { C7-C1-C6=135.620 } \\
\text { C8-C7-C1=105.408 } \\
\text { C9-C8-C7=123.095 } \\
\text { C10-C9-C8=117.147 } \\
\text { C11-C10-C9=120.301 } \\
\text { C12-C11-C10=122.380 } \\
\text { O13-C8-C7 =111.693 } \\
\mathrm{H} 14-C 3-C 2=121.190 \\
\mathrm{H} 15-C 4-C 3=119.194 \\
\mathrm{H} 16-C 5-C 4=119.361 \\
\mathrm{H} 17-C 6-C 5=120.641 \\
\mathrm{H} 18-C 9-C 8=121.356 \\
\mathrm{H} 19-\mathrm{C} 10-\mathrm{C} 9=120.215 \\
\mathrm{Cl} 20-\mathrm{C} 11-\mathrm{C} 10=118.650 \\
\mathrm{H} 21-\mathrm{Cl} 20-\mathrm{C} 11=120.598\end{array}$ & 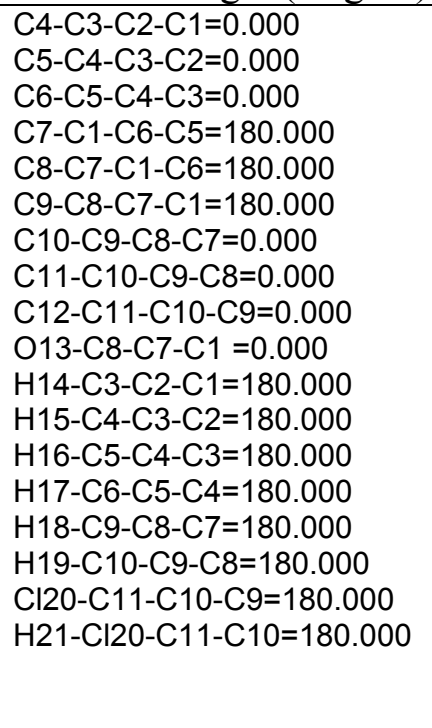 \\
\hline 2 & $\begin{array}{l}\mathrm{C} 2-\mathrm{C} 1=1.407 \\
\mathrm{C} 3-\mathrm{C} 2=1.387 \\
\mathrm{C} 4-\mathrm{C} 3=1.396 \\
\mathrm{C} 5-\mathrm{C} 4=1.405 \\
\mathrm{C} 6-\mathrm{C} 5=1.394 \\
\mathrm{C} 7-\mathrm{C} 1=1.453 \\
\mathrm{C} 8-\mathrm{C} 7=1.409 \\
\mathrm{C}-\mathrm{C} 8=1.390 \\
\mathrm{C} 10-\mathrm{C} 9=1.394 \\
\mathrm{C} 11-\mathrm{C} 10=1.403 \\
\mathrm{C} 12-\mathrm{C} 11=1.393 \\
\mathrm{O} 13-\mathrm{C} 8=1.369 \\
\mathrm{H} 14-\mathrm{C} 3=1.085\end{array}$ & $\begin{array}{l}\text { C3-C2-C1=123.449 } \\
C 4-C 3-C 2=116.551 \\
\text { C5-C4-C3=121.358 } \\
\text { C6-C5-C4=121.098 } \\
\text { C7-C1-C6=135.493 } \\
\text { C8-C7-C1=104.960 } \\
\text { C9-C8-C7=122.054 } \\
\text { C10-C9-C8=117.911 } \\
\text { C11-C10-C9=120.571 } \\
\text { C12-C11-C10=121.379 } \\
\text { O13-C8-C7 }=112.137 \\
\text { H14-C3-C2=121.231 } \\
\text { H15-C4-C3 }=119.186\end{array}$ & 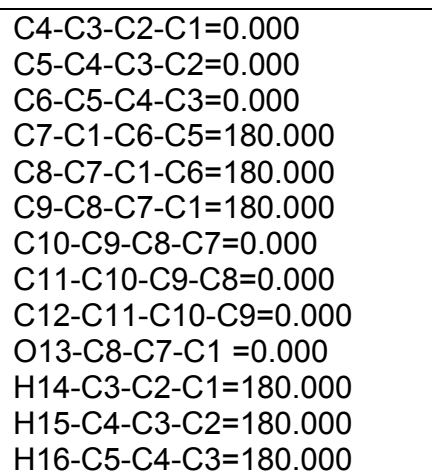 \\
\hline
\end{tabular}




\begin{tabular}{|c|c|c|c|}
\hline & $\begin{array}{l}\mathrm{H} 15-\mathrm{C} 4=1.086 \\
\mathrm{H} 16-\mathrm{C} 5=1.086 \\
\mathrm{H} 17-\mathrm{C} 6=1.086 \\
\mathrm{C} 18-\mathrm{C} 9=1.748 \\
\mathrm{H} 19-\mathrm{C} 10=1.085 \\
\mathrm{H} 20-\mathrm{C} 11=1.086 \\
\mathrm{H} 21-\mathrm{C} 12=1.086\end{array}$ & $\begin{array}{l}\mathrm{H} 16-\mathrm{C} 5-\mathrm{C} 4=119.353 \\
\mathrm{H} 17-\mathrm{C} 6-\mathrm{C} 5=120.641 \\
\mathrm{Cl} 18-\mathrm{C} 9-\mathrm{C} 8=120.776 \\
\mathrm{H} 19-\mathrm{C} 10-\mathrm{C} 9=118.997 \\
\mathrm{H} 20-\mathrm{C} 11-\mathrm{C} 10=118.815 \\
\mathrm{H} 21-\mathrm{Cl} 20-\mathrm{C} 11=120.682\end{array}$ & $\begin{array}{l}\text { H17-C6-C5-C4=180.000 } \\
\text { Cl18-C9-C8-C7=180.000 } \\
\text { H19-C10-C9-C8=180.000 } \\
\text { H20-C11-C10-C9=180.000 } \\
\text { H21-Cl20-C11-C10=180.000 }\end{array}$ \\
\hline 3 & $\begin{array}{l}\mathrm{C} 2-\mathrm{C} 1=1.409 \\
\mathrm{C} 3-\mathrm{C} 2=1.390 \\
\mathrm{C} 4-\mathrm{C} 3=1.394 \\
\mathrm{C} 5-\mathrm{C} 4=1.404 \\
\mathrm{C} 6-\mathrm{C} 5=1.393 \\
\mathrm{C} 7-\mathrm{C} 1=1.452 \\
\mathrm{C} 8-\mathrm{C} 7=1.406 \\
\mathrm{C} 9-\mathrm{C} 8=1.387 \\
\mathrm{C} 10-\mathrm{C} 9=1.395 \\
\mathrm{C} 11-\mathrm{C} 10=1.404 \\
\mathrm{C} 12-\mathrm{C} 11=1.392 \\
\mathrm{O} 13-\mathrm{C} 8=1.370 \\
\mathrm{Cl} 14-\mathrm{C} 3=1.747 \\
\mathrm{H} 15-\mathrm{C} 4=1.085 \\
\mathrm{H} 16-\mathrm{C} 5=1.086 \\
\mathrm{H} 17-\mathrm{C} 6=1.086 \\
\mathrm{H} 18-\mathrm{C} 9=1.084 \\
\mathrm{H} 19-\mathrm{C} 10=1.084 \\
\mathrm{Cl} 20-\mathrm{C} 11=1.761 \\
\mathrm{H} 21-\mathrm{C} 12=1.084\end{array}$ & $\begin{array}{l}\text { C3-C2-C1=122.057 } \\
\text { C4-C3-C2=117.826 } \\
\text { C5-C4-C3=120.643 } \\
\text { C6-C5-C4=121.391 } \\
\text { C7-C1-C6=135.431 } \\
\text { C8-C7-C1=105.522 } \\
\text { C9-C8-C7=123.215 } \\
\text { C10-C9-C8=117.007 } \\
\text { C11-C10-C9=120.378 } \\
\text { C12-C11-C10=122.366 } \\
\text { O13-C8-C7 =112.165 } \\
\text { Cl14-C3-C2=120.784 } \\
\text { H15-C4-C3=118.971 } \\
\text { H16-C5-C4=118.821 } \\
\text { H17-C6-C5=120.714 } \\
\text { H18-C9-C8=121.394 } \\
\text { H19-C10-C9=120.172 } \\
\text { Cl20-C11-C10=118.655 } \\
\text { H21-Cl20-C11=120.614 }\end{array}$ & 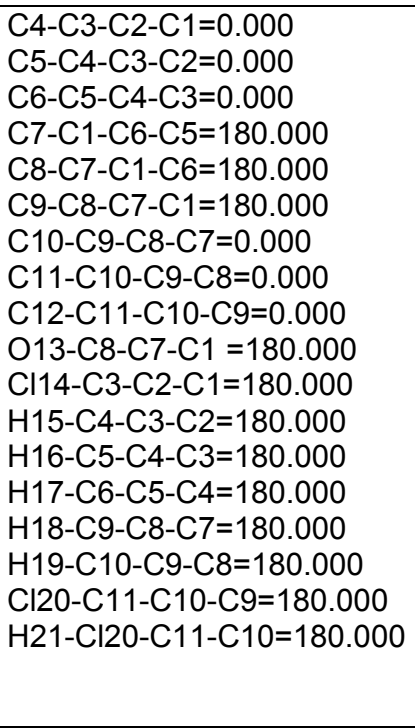 \\
\hline 4 & $\begin{array}{l}\mathrm{C} 2-\mathrm{C} 1=1.408 \\
\mathrm{C} 3-\mathrm{C} 2=1.388 \\
\mathrm{C} 4-\mathrm{C} 3=1.395 \\
\mathrm{C} 5-\mathrm{C} 4=1.404 \\
\mathrm{C} 6-\mathrm{C} 5=1.392 \\
\mathrm{C} 7-\mathrm{C} 1=1.452 \\
\mathrm{C} 8-\mathrm{C} 7=1.408 \\
\mathrm{C} 9-\mathrm{C} 8=1.388 \\
\mathrm{C} 10-\mathrm{C} 9=1.395 \\
\mathrm{C} 11-\mathrm{C} 10=1.404 \\
\mathrm{C} 12-\mathrm{C} 11=1.392 \\
\mathrm{O} 13-\mathrm{C} 8=1.376 \\
\mathrm{H} 14-\mathrm{C} 3=1.085 \\
\mathrm{H} 15-\mathrm{C} 4=1.084 \\
\mathrm{Cl} 16-\mathrm{C} 5=1.760 \\
\mathrm{H} 17-\mathrm{C} 6=1.085 \\
\mathrm{H} 18-\mathrm{C} 9=1.085 \\
\mathrm{H} 19-\mathrm{C} 10=1.084 \\
\mathrm{Cl} 20-\mathrm{C} 11=1.760 \\
\mathrm{H} 21-\mathrm{C} 12=1.085\end{array}$ & $\begin{array}{l}\text { C3-C2-C1=123.074 } \\
\text { C4-C3-C2=117.089 } \\
\text { C5-C4-C3=120.384 } \\
\text { C6-C5-C4=122.347 } \\
\text { C7-C1-C6=135.171 } \\
\text { C8-C7-C1=105.324 } \\
\text { C9-C8-C7=123.084 } \\
\text { C10-C9-C8=117.082 } \\
\text { C11-C10-C9=120.387 } \\
\text { C12-C11-C10=122.347 } \\
\text { O13-C8-C7 =111.725 } \\
\mathrm{H} 14-C 3-C 2=121.377 \\
\mathrm{H} 15-C 4-C 3=120.184 \\
\mathrm{Cl} 16-C 5-C 4=118.656 \\
\mathrm{H} 17-C 6-C 5=120.604 \\
\mathrm{H} 18-C 9-C 8=121.377 \\
\mathrm{H} 19-\mathrm{C} 10-\mathrm{C} 9=120.182 \\
\mathrm{Cl} 20-\mathrm{C} 11-\mathrm{C} 10=118.651 \\
\mathrm{H} 21-\mathrm{Cl} 20-\mathrm{C} 11=120.610\end{array}$ & $\begin{array}{l}\text { C4-C3-C2-C1=0.000 } \\
\text { C5-C4-C3-C2=0.000 } \\
\text { C6-C5-C4-C3=0.000 } \\
\text { C7-C1-C6-C5=180.000 } \\
\text { C8-C7-C1-C6=180.000 } \\
\text { C9-C8-C7-C1=180.000 } \\
\text { C10-C9-C8-C7=0.000 } \\
\text { C11-C10-C9-C8=0.000 } \\
\text { C12-C11-C10-C9=0.000 } \\
\text { O13-C8-C7-C1 =180.000 } \\
\text { H14-C3-C2-C1=180.000 } \\
\text { H15-C4-C3-C2=180.000 } \\
\text { Cl16-C5-C4-C3=180.000 } \\
\text { H17-C6-C5-C4=180.000 } \\
\text { H18-C9-C8-C7=180.000 } \\
\text { H19-C10-C9-C8=180.000 } \\
\text { Cl20-C11-C10-C9=180.000 } \\
\text { H21-Cl20-C11-C10=180.000 }\end{array}$ \\
\hline 5 & $\begin{array}{l}\mathrm{C} 2-\mathrm{C} 1=1.409 \\
\mathrm{C} 3-\mathrm{C} 2=1.389 \\
\mathrm{C} 4-\mathrm{C} 3=1.394 \\
\mathrm{C} 5-\mathrm{C} 4=1.403 \\
\mathrm{C} 6-\mathrm{C} 5=1.393 \\
\mathrm{C} 7-\mathrm{C} 1=1.452 \\
\mathrm{C} 8-\mathrm{C} 7=1.410 \\
\mathrm{C} 9-\mathrm{C} 8=1.386 \\
\mathrm{C} 10-\mathrm{C} 9=1.394 \\
\mathrm{C} 11-\mathrm{C} 10=1.402 \\
\mathrm{C} 12-\mathrm{C} 11=1.392 \\
\mathrm{O} 13-\mathrm{C} 8=1.372 \\
\mathrm{Cl} 14-\mathrm{C} 3=1.746 \\
\mathrm{H} 15-\mathrm{C} 4=1.085 \\
\mathrm{H} 16-\mathrm{C} 5=1.086 \\
\mathrm{H} 17-\mathrm{C} 6=1.084 \\
\mathrm{H} 18-\mathrm{C} 9=1.083 \\
\mathrm{C} 19-\mathrm{C} 10=1.754\end{array}$ & 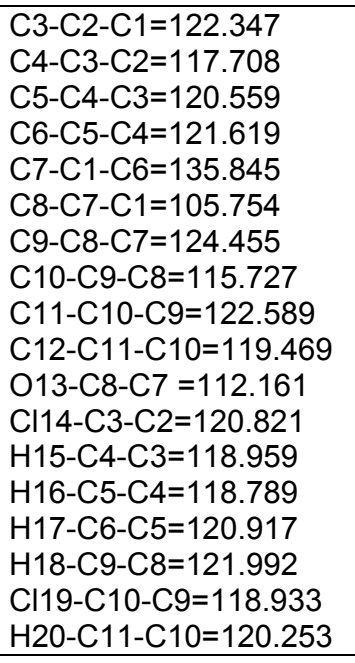 & 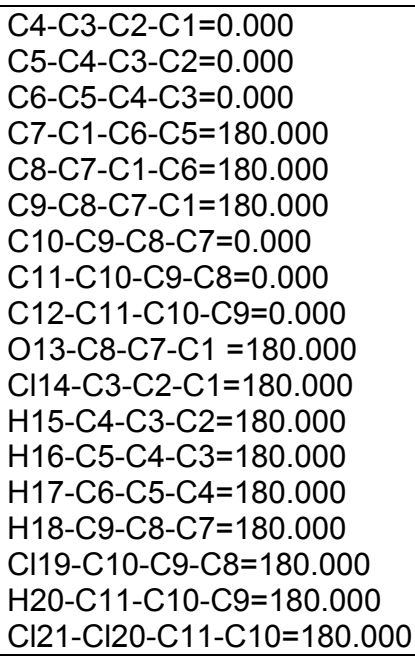 \\
\hline
\end{tabular}




\begin{tabular}{|c|c|c|c|}
\hline & $\begin{array}{l}\mathrm{H} 20-\mathrm{C} 11=1.083 \\
\mathrm{Cl} 21-\mathrm{C} 12=1.752\end{array}$ & $\mathrm{Cl} 21-\mathrm{Cl} 20-\mathrm{C} 11=119.0577$ & \\
\hline 6 & $\begin{array}{l}\mathrm{C} 2-\mathrm{C} 1=1.408 \\
\mathrm{C} 3-\mathrm{C} 2=1.386 \\
\mathrm{C} 4-\mathrm{C} 3=1.395 \\
\mathrm{C} 5-\mathrm{C} 4=1.403 \\
\mathrm{C} 6-\mathrm{C} 5=1.392 \\
\mathrm{C} 7-\mathrm{C} 1=1.451 \\
\mathrm{C} 8-\mathrm{C} 7=1.410 \\
\mathrm{C} 9-\mathrm{C} 8=1.387 \\
\mathrm{C} 10-\mathrm{C} 9=1.394 \\
\mathrm{C} 11-\mathrm{C} 10=1.402 \\
\mathrm{C} 12-\mathrm{C} 11=1.392 \\
\mathrm{O} 13-\mathrm{C} 8=1.370 \\
\mathrm{H} 14-\mathrm{C} 3=1.084 \\
\mathrm{H} 15-\mathrm{C} 4=1.084 \\
\mathrm{Cl} 16-\mathrm{C} 5=1.760 \\
\mathrm{H} 17-\mathrm{C} 6=1.083 \\
\mathrm{H} 18-\mathrm{C} 9=1.083 \\
\mathrm{Cl} 19-\mathrm{C} 10=1.754 \\
\mathrm{H} 20-\mathrm{C} 11=1.083 \\
\mathrm{Cl} 21-\mathrm{C} 12=1.752\end{array}$ & $\begin{array}{l}\text { C3-C2-C1=123.313 } \\
\text { C4-C3-C2=117.010 } \\
\text { C5-C4-C3=120.322 } \\
\text { C6-C5-C4=122.507 } \\
\text { C7-C1-C6=135.581 } \\
\text { C8-C7-C1=105.569 } \\
\text { C9-C8-C7=124.267 } \\
\text { C10-C9-C8 }=115.797 \\
\text { C11-C10-C9=122.647 } \\
\text { C12-C11-C10=119.409 } \\
\text { O13-C8-C7 =111.483 } \\
\text { H14-C3-C2=121.335 } \\
\mathrm{H} 15-C 4-C 3=120.195 \\
\text { Cl16-C5-C4=118.596 } \\
\mathrm{H} 17-C 6-C 5=120.840 \\
\mathrm{H} 18-C 9-C 8=122.011 \\
\mathrm{Cl} 19-\mathrm{C} 10-\mathrm{C} 9=118.890 \\
\mathrm{H} 20-\mathrm{C} 11-\mathrm{C} 10=120.280 \\
\mathrm{Cl} 21-\mathrm{H} 20-\mathrm{C} 11=119.135\end{array}$ & $\begin{array}{l}\mathrm{C} 4-\mathrm{C} 3-\mathrm{C} 2-\mathrm{C} 1=0.000 \\
\mathrm{C} 5-\mathrm{C} 4-\mathrm{C} 3-\mathrm{C} 2=0.000 \\
\mathrm{C} 6-\mathrm{C} 5-\mathrm{C} 4-\mathrm{C} 3=0.000 \\
\mathrm{C} 7-\mathrm{C} 1-\mathrm{C} 6-\mathrm{C} 5=180.000 \\
\mathrm{C} 8-\mathrm{C} 7-\mathrm{C} 1-\mathrm{C} 6=180.000 \\
\mathrm{C} 9-\mathrm{C} 8-\mathrm{C} 7-\mathrm{C} 1=180.000 \\
\mathrm{C} 10-\mathrm{C} 9-\mathrm{C} 8-\mathrm{C} 7=0.000 \\
\mathrm{C} 11-\mathrm{C} 10-\mathrm{C} 9-\mathrm{C} 8=0.000 \\
\mathrm{C} 12-\mathrm{C} 11-\mathrm{C} 10-\mathrm{C} 9=0.000 \\
\mathrm{O} 13-\mathrm{C} 8-\mathrm{C} 7-\mathrm{C} 1=0.000 \\
\mathrm{H} 14-\mathrm{C} 3-\mathrm{C} 2-\mathrm{C} 1=180.000 \\
\mathrm{H} 15-\mathrm{C} 4-\mathrm{C} 3-\mathrm{C} 2=180.000 \\
\mathrm{Cl} 16-\mathrm{C} 5-\mathrm{C} 4-\mathrm{C} 3=180.000 \\
\mathrm{H} 17-\mathrm{C} 6-\mathrm{C} 5-\mathrm{C} 4=180.000 \\
\mathrm{H} 18-\mathrm{C} 9-\mathrm{C} 8-\mathrm{C} 7=180.000 \\
\mathrm{Cl} 19-\mathrm{C} 10-\mathrm{C} 9-\mathrm{C} 8=180.000 \\
\mathrm{H} 20-\mathrm{C} 11-\mathrm{C} 10-\mathrm{C} 9=180.000 \\
\mathrm{Cl} 21-\mathrm{H} 20-\mathrm{C} 11-\mathrm{C} 10=180.000\end{array}$ \\
\hline 7 & $\begin{array}{l}\mathrm{C} 2-\mathrm{C} 1=1.406 \\
\mathrm{C} 3-\mathrm{C} 2=1.387 \\
\mathrm{C} 4-\mathrm{C} 3=1.396 \\
\mathrm{C} 5-\mathrm{C} 4=1.405 \\
\mathrm{C} 6-\mathrm{C} 5=1.393 \\
\mathrm{C} 7-\mathrm{C} 1=1.452 \\
\mathrm{C} 8-\mathrm{C} 7=1.406 \\
\mathrm{C} 9-\mathrm{C} 8=1.390 \\
\mathrm{C} 10-\mathrm{C} 9=1.406 \\
\mathrm{C} 11-\mathrm{C} 10=1.414 \\
\mathrm{C} 12-\mathrm{C} 11=1.392 \\
\mathrm{O} 13-\mathrm{C} 8=1.367 \\
\mathrm{H} 14-\mathrm{C} 3=1.085 \\
\mathrm{H} 15-\mathrm{C} 4=1.086 \\
\mathrm{H} 16-\mathrm{C} 5=1.086 \\
\mathrm{H} 17-\mathrm{C} 6=1.0864 \\
\mathrm{Cl} 18-\mathrm{C} 9=1.736 \\
\mathrm{Cl} 19-\mathrm{C} 10=1.737 \\
\mathrm{Cl} 20-\mathrm{C} 11=1.749 \\
\mathrm{H} 21-\mathrm{C} 12=1.084\end{array}$ & $\begin{array}{l}\text { C3-C2-C1=123.479 } \\
\text { C4-C3-C2=116.451 } \\
\text { C5-C4-C3=121.421 } \\
\text { C6-C5-C4=121.131 } \\
\text { C7-C1-C6=135.586 } \\
\text { C8-C7-C1=105.190 } \\
\text { C9-C8-C7=122.761 } \\
\text { C10-C9-C8=117.660 } \\
\text { C11-C10-C9=119.799 } \\
\text { C12-C11-C10=121.676 } \\
\text { O13-C8-C7 =112.133 } \\
\text { H14-C3-C2=121.273 } \\
\text { H15-C4-C3=119.163 } \\
\text { H16-C5-C4=119.337 } \\
\text { H17-C6-C5=120.689 } \\
\text { Cl18-C9-C8=119.603 } \\
\text { Cl19-C10-C9=119.653 } \\
\text { Cl20-C11-C10 =120.384 } \\
\text { H21-Cl20-C11=119.604 }\end{array}$ & $\begin{array}{l}\text { C4-C3-C2-C1=0.000 } \\
\text { C5-C4-C3-C2=0.000 } \\
\text { C6-C5-C4-C3=0.000 } \\
\text { C7-C1-C6-C5=180.000 } \\
\text { C8-C7-C1-C6=180.000 } \\
\text { C9-C8-C7-C1=180.000 } \\
\text { C10-C9-C8-C7=0.000 } \\
\text { C11-C10-C9-C8=0.000 } \\
\text { C12-C11-C10-C9=0.000 } \\
\text { O13-C8-C7-C1 =0.000 } \\
\text { H14-C3-C2-C1=180.000 } \\
\text { H15-C4-C3-C2=180.000 } \\
\text { H16-C5-C4-C3=180.000 } \\
\text { H17-C6-C5-C4=180.000 } \\
\text { Cl18-C9-C8-C7=180.000 } \\
\text { Cl19-C10-C9-C8=180.000 } \\
\text { Cl20-C11-C10-C9=180.000 } \\
\text { H21-Cl20-C11-C10=180.000 }\end{array}$ \\
\hline 8 & $\begin{array}{l}\mathrm{C} 2-\mathrm{C} 1=1.407 \\
\mathrm{C} 3-\mathrm{C} 2=1.387 \\
\mathrm{C} 4-\mathrm{C} 3=1.395 \\
\mathrm{C} 5-\mathrm{C} 4=1.404 \\
\mathrm{C} 6-\mathrm{C} 5=1.392 \\
\mathrm{C} 7-\mathrm{C} 1=1.451 \\
\mathrm{C} 8-\mathrm{C} 7=1.407 \\
\mathrm{C} 9-\mathrm{C} 8=1.385 \\
\mathrm{C} 10-\mathrm{C} 9=1.397 \\
\mathrm{C} 11-\mathrm{C} 10=1.412 \\
\mathrm{C} 12-\mathrm{C} 11=1.394 \\
\mathrm{O} 13-\mathrm{C} 8=1.373 \\
\mathrm{H} 14-\mathrm{C} 3=1.084 \\
\mathrm{H} 15-\mathrm{C} 4=1.084 \\
\mathrm{Cl} 16-\mathrm{C} 5=1.759 \\
\mathrm{H} 17-\mathrm{C} 6=1.084 \\
\mathrm{H} 18-\mathrm{C} 9=1.083 \\
\mathrm{Cl} 19-\mathrm{C} 10=1.746 \\
\mathrm{Cl} 20-\mathrm{C} 11=1.749 \\
\mathrm{Cl} 21-\mathrm{C} 12=1.084\end{array}$ & 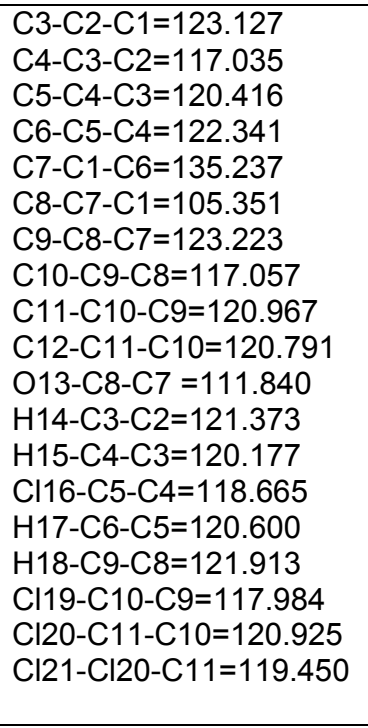 & $\begin{array}{l}\mathrm{C} 4-\mathrm{C} 3-\mathrm{C} 2-\mathrm{C} 1=0.000 \\
\mathrm{C} 5-\mathrm{C} 4-\mathrm{C} 3-\mathrm{C} 2=0.000 \\
\mathrm{C} 6-\mathrm{C} 5-\mathrm{C} 4-\mathrm{C} 3=0.000 \\
\mathrm{C} 7-\mathrm{C} 1-\mathrm{C} 6-\mathrm{C} 5=180.000 \\
\mathrm{C} 8-\mathrm{C} 7-\mathrm{C} 1-\mathrm{C} 6=180.000 \\
\mathrm{C} 9-\mathrm{C} 8-\mathrm{C} 7-\mathrm{C} 1=180.000 \\
\mathrm{C} 10-\mathrm{C} 9-\mathrm{C} 8-\mathrm{C} 7=0.00 \\
\mathrm{C} 11-\mathrm{C} 10-\mathrm{C} 9-\mathrm{C} 8=0.00 \\
\mathrm{C} 12-\mathrm{C} 11-\mathrm{C} 10-\mathrm{C} 9=0.00 \\
\mathrm{O} 13-\mathrm{C} 8-\mathrm{C} 7-\mathrm{C} 1=0.00 \\
\mathrm{H} 14-\mathrm{C} 3-\mathrm{C} 2-\mathrm{C} 1=180.000 \\
\mathrm{H} 15-\mathrm{C} 4-\mathrm{C} 3-\mathrm{C} 2=180.000 \\
\mathrm{Cl} 16-\mathrm{C} 5-\mathrm{C} 4-\mathrm{C} 3=180.000 \\
\mathrm{H} 17-\mathrm{C} 6-\mathrm{C} 5-\mathrm{C} 4=180.000 \\
\mathrm{H} 18-\mathrm{C} 9-\mathrm{C} 8-\mathrm{C} 7=180.000 \\
\mathrm{Cl} 19-\mathrm{C} 10-\mathrm{C} 9-\mathrm{C} 8=180.000 \\
\mathrm{Cl} 20-\mathrm{C} 11-\mathrm{C} 10-\mathrm{C} 9=180.000 \\
\mathrm{Cl} 21-\mathrm{Cl} 20-\mathrm{C} 11-\mathrm{C} 10=180.000\end{array}$ \\
\hline 9 & $\begin{array}{l}C 2-C 1=1.407 \\
C 3-C 2=1.390 \\
C 4-C 3=1.395\end{array}$ & $\begin{array}{l}C 3-C 2-C 1=122.259 \\
C 4-C 3-C 2=117.573 \\
\text { C5-C4-C3=120.742 }\end{array}$ & $\begin{array}{l}\text { C4-C3-C2-C1 }=0.000 \\
\text { C5-C4-C3-C2 }=0.000 \\
\text { C6-C5-C4-C3 }=0.000\end{array}$ \\
\hline
\end{tabular}




\begin{tabular}{|c|c|c|c|}
\hline & $\begin{array}{l}\mathrm{C} 5-\mathrm{C} 4=1.404 \\
\mathrm{C} 6-\mathrm{C} 5=1.393 \\
\mathrm{C} 7-\mathrm{C} 1=1.452 \\
\mathrm{C} 8-\mathrm{C} 7=1.404 \\
\mathrm{C} 9-\mathrm{C} 8=1.390 \\
\mathrm{C} 10-\mathrm{C} 9=1.406 \\
\mathrm{C} 11-\mathrm{C} 10=1.414 \\
\mathrm{C} 12-\mathrm{C} 11=1.392 \\
\mathrm{O} 13-\mathrm{C} 8=1.369 \\
\mathrm{Cl} 14-\mathrm{C} 3=1.744 \\
\mathrm{H} 15-\mathrm{C} 4=1.085 \\
\mathrm{H} 16-\mathrm{C} 5=1.086 \\
\mathrm{H} 17-\mathrm{C} 6=1.086 \\
\mathrm{Cl} 18-\mathrm{C} 9=1.734 \\
\mathrm{Cl} 19-\mathrm{C} 10=1.736 \\
\mathrm{Cl} 20-\mathrm{C} 11=1.749 \\
\mathrm{H} 21-\mathrm{C} 12=1.084\end{array}$ & $\begin{array}{l}\text { C6-C5-C4=121.451 } \\
\text { C7-C1-C6=135.365 } \\
\text { C8-C7-C1=105.299 } \\
\text { C9-C8-C7=122.897 } \\
\text { C10-C9-C8=117.496 } \\
\text { C11-C10-C9=119.865 } \\
\text { C12-C11-C10=121.688 } \\
\text { O13-C8-C7 =112.090 } \\
\text { Cl14-C3-C2=120.879 } \\
\text { H15-C4-C3=118.909 } \\
\text { H16-C5-C4=118.795 } \\
\text { H17-C6-C5=120.781 } \\
\text { Cl18-C9-C8=119.666 } \\
\text { Cl19-C10-C9=119.605 } \\
\text { Cl20-C11-C10=120.384 } \\
\text { H21-Cl20-C11=119.638 }\end{array}$ & $\begin{array}{l}\text { C7-C1-C6-C5=180.000 } \\
\text { C8-C7-C1-C6=180.000 } \\
\text { C9-C8-C7-C1=180.000 } \\
\text { C10-C9-C8-C7=0.000 } \\
\text { C11-C10-C9-C8 }=0.000 \\
\text { C12-C11-C10-C9=0.000 } \\
\text { O13-C8-C7-C1 =0.000 } \\
\text { Cl14-C3-C2-C1=180.000 } \\
\text { H15-C4-C3-C2=180.000 } \\
\text { H16-C5-C4-C3=180.000 } \\
\text { H17-C6-C5-C4=180.000 } \\
\text { Cl18-C9-C8-C7=180.000 } \\
\text { Cl19-C10-C9-C8=180.000 } \\
\text { Cl20-C11-C10-C9=180.000 } \\
\text { H21-Cl20-C11-C10=180.000 }\end{array}$ \\
\hline 10 & $\begin{array}{l}\mathrm{C} 2-\mathrm{C} 1=1.406 \\
\mathrm{C} 3-\mathrm{C} 2=1.387 \\
\mathrm{C} 4-\mathrm{C} 3=1.395 \\
\mathrm{C} 5-\mathrm{C} 4=1.404 \\
\mathrm{C} 6-\mathrm{C} 5=1.392 \\
\mathrm{C} 7-\mathrm{C} 1=1.452 \\
\mathrm{C} 8-\mathrm{C} 7=1.405 \\
\mathrm{C} 9-\mathrm{C} 8=1.390 \\
\mathrm{C} 10-\mathrm{C} 9=1.405 \\
\mathrm{C} 11-\mathrm{C} 10=1.415 \\
\mathrm{C} 12-\mathrm{C} 11=1.392 \\
\mathrm{O} 13-\mathrm{C} 8=1.367 \\
\mathrm{H} 14-\mathrm{C} 3=1.084 \\
\mathrm{H} 15-\mathrm{C} 4=1.084 \\
\mathrm{Cl} 16-\mathrm{C} 5=1.758 \\
\mathrm{H} 17-\mathrm{C} 6=1.084 \\
\mathrm{Cl} 18-\mathrm{C} 9=1.735 \\
\mathrm{Cl} 19-\mathrm{C} 10=1.736 \\
\mathrm{Cl} 20-\mathrm{C} 11=1.748 \\
\mathrm{H} 21-\mathrm{C} 12=1.084\end{array}$ & $\begin{array}{l}\text { C3-C2-C1=123.228 } \\
\text { C4-C3-C2=116.920 } \\
\text { C5-C4-C3=120.470 } \\
\text { C6-C5-C4=122.338 } \\
\text { C7-C1-C6=135.138 } \\
\text { C8-C7-C1=105.103 } \\
\text { C9-C8-C7=122.770 } \\
\text { C10-C9-C8=117.592 } \\
\text { C11-C10-C9=119.857 } \\
\text { C12-C11-C10=121.663 } \\
\text { O13-C8-C7 =112.171 } \\
\text { H14-C3-C2=121.426 } \\
\text { H15-C4-C3=120.144 } \\
\text { Cl16-C5-C4=118.670 } \\
\text { H17-C6-C5=120.619 } \\
\text { Cl18-C9-C8=119.593 } \\
\text { Cl19-C10-C9=119.625 } \\
\text { Cl20-C11-C10=120.391 } \\
\text { H21-Cl20-C11=119.627 }\end{array}$ & 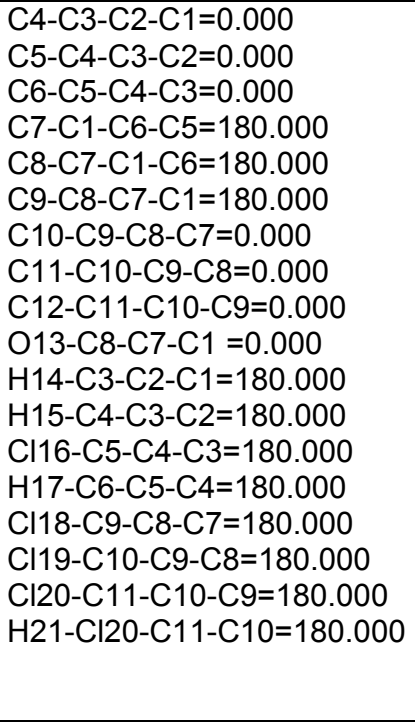 \\
\hline 11 & $\begin{array}{l}\mathrm{C} 2-\mathrm{C} 1=1.408 \\
\mathrm{C} 3-\mathrm{C} 2=1.390 \\
\mathrm{C} 4-\mathrm{C} 3=1.394 \\
\mathrm{C} 5-\mathrm{C} 4=1.402 \\
\mathrm{C} 6-\mathrm{C} 5=1.399 \\
\mathrm{C} 7-\mathrm{C} 1=1.451 \\
\mathrm{C} 8-\mathrm{C} 7=1.410 \\
\mathrm{C} 9-\mathrm{C} 8=1.386 \\
\mathrm{C} 10-\mathrm{C} 9=1.394 \\
\mathrm{C} 11-\mathrm{C} 10=1.403 \\
\mathrm{C} 12-\mathrm{C} 11=1.392 \\
\mathrm{O} 13-\mathrm{C} 8=1.370 \\
\mathrm{Cl} 14-\mathrm{C} 3=1.743 \\
\mathrm{H} 15-\mathrm{C} 4=1.083 \\
\mathrm{Cl} 16-\mathrm{C} 5=1.757 \\
\mathrm{H} 17-\mathrm{C} 6=1.082 \\
\mathrm{H} 18-\mathrm{C} 9=1.083 \\
\mathrm{Cl} 19-\mathrm{C} 10=1.752 \\
\mathrm{H} 20-\mathrm{C} 11=1.083 \\
\mathrm{Cl} 21-\mathrm{C} 12=1.751\end{array}$ & $\begin{array}{l}\text { C3-C2-C1=122.169 } \\
\text { C4-C3-C2=118.018 } \\
\text { C5-C4-C3=119.762 } \\
\text { C6-C5-C4=119.936 } \\
\text { C7-C1-C6=135.388 } \\
\text { C8-C7-C1=105.693 } \\
\text { C9-C8-C7=124.417 } \\
\text { C10-C9-C8=115.687 } \\
\text { C11-C10-C9=122.625 } \\
\text { C12-C11-C10=119.514 } \\
\text { O13-C8-C7 =112.223 } \\
\text { Cl14-C3-C2=120.880 } \\
\text { H15-C4-C3=119.876 } \\
\text { Cl16-C5-C4=118.250 } \\
\text { H17-C6-C5=121.718 } \\
\text { H18-C9-C8=122.020 } \\
\text { Cl19-C10-C9=118.923 } \\
\text { H20-C11-C10 }=120.219 \\
\text { Cl21-H20-C11=119.166 }\end{array}$ & $\begin{array}{l}\text { C4-C3-C2-C1=0.000 } \\
\text { C5-C4-C3-C2=0.000 } \\
\text { C6-C5-C4-C3=0.000 } \\
\text { C7-C1-C6-C5=180.000 } \\
\text { C8-C7-C1-C6=180.000 } \\
\text { C9-C8-C7-C1=180.000 } \\
\text { C10-C9-C8-C7=0.000 } \\
\text { C11-C10-C9-C8=0.000 } \\
\text { C12-C11-C10-C9=0.000 } \\
\text { O13-C8-C7-C1 =180.000 } \\
\text { Cl14-C3-C2-C1=180.000 } \\
\text { H15-C4-C3-C2=180.000 } \\
\text { Cl16-C5-C4-C3=180.000 } \\
\text { H17-C6-C5-C4=180.000 } \\
\text { H18-C9-C8-C7=180.000 } \\
\text { Cl19-C10-C9-C8=180.000 } \\
\text { H20-C11-C10-C9=180.000 } \\
\text { Cl21-H20-C11-C10=180.000 }\end{array}$ \\
\hline 12 & $\begin{array}{l}C 2-C 1=1.407 \\
C 3-C 2=1.384 \\
C 4-C 3=1.397 \\
C 5-C 4=1.412 \\
C 6-C 5=1.394 \\
C 7-C 1=1.451 \\
C 8-C 7=1.407 \\
C 9-C 8=1.384\end{array}$ & $\begin{array}{l}\text { C3-C2-C1=123.245 } \\
\text { C4-C3-C2 }=117.008 \\
\text { C5-C4-C3 }=120.997 \\
\text { C6-C5-C4=120.787 } \\
\text { C7-C1-C6 }=135.621 \\
\text { C8-C7-C1 }=105.289 \\
\text { C9-C8-C7=123.284 } \\
\text { C10-C9-C8 }=117.006\end{array}$ & $\begin{array}{l}\text { C4-C3-C2-C1 }=0.000 \\
\text { C5-C4-C3-C2 }=0.000 \\
\text { C6-C5-C4-C3 }=0.000 \\
\text { C7-C1-C6-C5 }=180.000 \\
\text { C8-C7-C1-C6 }=180.000 \\
\text { C9-C8-C7-C1 }=180.000 \\
\text { C10-C9-C8-C7 }=0.000 \\
\text { C11-C10-C9-C8 }=0.000\end{array}$ \\
\hline
\end{tabular}




\begin{tabular}{|c|c|c|c|}
\hline & $\begin{array}{l}\mathrm{C} 10-\mathrm{C} 9=1.397 \\
\mathrm{C} 11-\mathrm{C} 10=1.412 \\
\mathrm{C} 12-\mathrm{C} 11=1.394 \\
\mathrm{O} 13-\mathrm{C} 8=1.375 \\
\mathrm{H} 14-\mathrm{C} 3=1.083 \\
\mathrm{Cl} 15-\mathrm{C} 4=1.745 \\
\mathrm{Cl} 16-\mathrm{C} 5=1.748 \\
\mathrm{H} 17-\mathrm{C} 6=1.084 \\
\mathrm{H} 18-\mathrm{C} 9=1.083 \\
\mathrm{Cl} 19-\mathrm{C} 10=1.745 \\
\mathrm{Cl} 20-\mathrm{C} 11=1.748 \\
\mathrm{H} 21-\mathrm{C} 12=1.084\end{array}$ & $\begin{array}{l}\mathrm{C} 11-\mathrm{C} 10-\mathrm{C} 9=120.974 \\
\mathrm{C} 12-\mathrm{C} 11-\mathrm{C} 10=120.803 \\
\mathrm{O} 13-\mathrm{C} 8-\mathrm{C} 7=111.837 \\
\mathrm{H} 14-\mathrm{C} 3-\mathrm{C} 2=121.982 \\
\mathrm{Cl} 15-\mathrm{C} 4-\mathrm{C} 3=117.969 \\
\mathrm{Cl} 16-\mathrm{C} 5-\mathrm{C} 4=120.931 \\
\mathrm{H} 17-\mathrm{C} 6-\mathrm{C} 5=119.475 \\
\mathrm{H} 18-\mathrm{C} 9-\mathrm{C} 8=121.949 \\
\mathrm{Cl} 19-\mathrm{C} 10-\mathrm{C} 9=117.981 \\
\mathrm{Cl} 20-\mathrm{C} 11-\mathrm{C} 10=120.919 \\
\mathrm{H} 21-\mathrm{Cl} 20-\mathrm{C} 11=119.460\end{array}$ & $\begin{array}{l}\mathrm{C} 12-\mathrm{C} 11-\mathrm{C} 10-\mathrm{C} 9=0.000 \\
\mathrm{O} 13-\mathrm{C} 8-\mathrm{C} 7-\mathrm{C} 1=180.000 \\
\mathrm{H} 14-\mathrm{C} 3-\mathrm{C} 2-\mathrm{C} 1=180.000 \\
\mathrm{Cl} 15-\mathrm{C} 4-\mathrm{C} 3-\mathrm{C} 2=180.000 \\
\mathrm{Cl} 16-\mathrm{C} 5-\mathrm{C} 4-\mathrm{C} 3=180.000 \\
\mathrm{H} 17-\mathrm{C} 6-\mathrm{C} 5-\mathrm{C} 4=180.000 \\
\mathrm{H} 18-\mathrm{C} 9-\mathrm{C} 8-\mathrm{C} 7=180.000 \\
\mathrm{Cl} 19-\mathrm{C} 10-\mathrm{C} 9-\mathrm{C} 8=180.000 \\
\mathrm{Cl} 20-\mathrm{C} 11-\mathrm{C} 10-\mathrm{C} 9=180.000 \\
\mathrm{H} 21-\mathrm{Cl} 20-\mathrm{C} 11-\mathrm{C} 10=180.000\end{array}$ \\
\hline 13 & $\begin{array}{l}\mathrm{C} 2-\mathrm{C} 1=1.407 \\
\mathrm{C} 3-\mathrm{C} 2=1.387 \\
\mathrm{C} 4-\mathrm{C} 3=1.394 \\
\mathrm{C} 5-\mathrm{C} 4=1.404 \\
\mathrm{C} 6-\mathrm{C} 5=1.392 \\
\mathrm{C} 7-\mathrm{C} 1=1.453 \\
\mathrm{C} 8-\mathrm{C} 7=1.409 \\
\mathrm{C} 9-\mathrm{C} 8=1.390 \\
\mathrm{C} 10-\mathrm{C} 9=1.391 \\
\mathrm{C} 11-\mathrm{C} 10=1.403 \\
\mathrm{C} 12-\mathrm{C} 11=1.400 \\
\mathrm{O} 13-\mathrm{C} 8=1.365 \\
\mathrm{H} 14-\mathrm{C} 3=1.084 \\
\mathrm{H} 15-\mathrm{C} 4=1.084 \\
\mathrm{Cl} 16-\mathrm{C} 5=1.759 \\
\mathrm{H} 17-\mathrm{C} 6=1.082 \\
\mathrm{Cl} 18-\mathrm{C} 9=1.741 \\
\mathrm{H} 19-\mathrm{C} 10=1.083 \\
\mathrm{Cl} 20-\mathrm{C} 11=1.747 \\
\mathrm{Cl} 21-\mathrm{C} 12=1.741\end{array}$ & $\begin{array}{l}\text { C3-C2-C1=123.446 } \\
\text { C4-C3-C2=116.926 } \\
\text { C5-C4-C3=120.349 } \\
\text { C6-C5-C4=122.518 } \\
\text { C7-C1-C6=135.578 } \\
\text { C8-C7-C1=105.065 } \\
\text { C9-C8-C7=122.462 } \\
\text { C10-C9-C8=117.788 } \\
\text { C11-C10-C9=120.800 } \\
\text { C12-C11-C10=121.205 } \\
\text { O13-C8-C7 =112.087 } \\
\mathrm{H} 14-C 3-C 2=121.328 \\
\mathrm{H} 15-C 4-C 3=120.189 \\
\mathrm{Cl} 16-\mathrm{C} 5-\mathrm{C} 4=118.591 \\
\mathrm{H} 17-\mathrm{C} 6-\mathrm{C} 5=120.776 \\
\mathrm{Cl} 18-\mathrm{C} 9-\mathrm{C} 8=121.023 \\
\mathrm{H} 19-\mathrm{C} 10-\mathrm{C} 9=119.834 \\
\mathrm{Cl} 20-\mathrm{C} 11-\mathrm{C} 10=117.754 \\
\mathrm{Cl} 21-\mathrm{Cl} 20-\mathrm{C} 11=121.527\end{array}$ & $\begin{array}{l}\text { C4-C3-C2-C1=0.000 } \\
\text { C5-C4-C3-C2=0.000 } \\
\text { C6-C5-C4-C3=0.000 } \\
\text { C7-C1-C6-C5=180.000 } \\
\text { C8-C7-C1-C6=180.000 } \\
\text { C9-C8-C7-C1=180.000 } \\
\text { C10-C9-C8-C7=0.000 } \\
\text { C11-C10-C9-C8=0.000 } \\
\text { C12-C11-C10-C9=0.000 } \\
\text { O13-C8-C7-C1 =0.000 } \\
\text { H14-C3-C2-C1=180.000 } \\
\text { H15-C4-C3-C2=180.000 } \\
\text { Cl16-C5-C4-C3=180.000 } \\
\text { H17-C6-C5-C4=180.000 } \\
\text { Cl18-C9-C8-C7=180.000 } \\
\text { H19-C10-C9-C8=180.000 } \\
\text { Cl20-C11-C10-C9=180.000 } \\
\text { Cl21-Cl20-C11-C10=180.000 }\end{array}$ \\
\hline 14 & $\begin{array}{l}\mathrm{C} 2-\mathrm{C} 1=1.406 \\
\mathrm{C} 3-\mathrm{C} 2=1.393 \\
\mathrm{C} 4-\mathrm{C} 3=1.401 \\
\mathrm{C} 5-\mathrm{C} 4=1.406 \\
\mathrm{C} 6-\mathrm{C} 5=1.389 \\
\mathrm{C} 7-\mathrm{C} 1=1.453 \\
\mathrm{C} 8-\mathrm{C} 7=1.408 \\
\mathrm{C} 9-\mathrm{C} 8=1.389 \\
\mathrm{C} 10-\mathrm{C} 9=1.392 \\
\mathrm{C} 11-\mathrm{C} 10=1.403 \\
\mathrm{C} 12-\mathrm{C} 11=1.400 \\
\mathrm{O} 13-\mathrm{C} 8=1.367 \\
\mathrm{Cl} 14-\mathrm{C} 3=1.734 \\
\mathrm{Cl} 15-\mathrm{C} 4=1.744 \\
\mathrm{H} 16-\mathrm{C} 5=1.084 \\
\mathrm{H} 17-\mathrm{C} 6=1.083 \\
\mathrm{Cl} 18-\mathrm{C} 9=1.740 \\
\mathrm{H} 19-\mathrm{C} 10=1.083 \\
\mathrm{Cl} 20-\mathrm{C} 11=1.746 \\
\mathrm{Cl} 21-\mathrm{C} 12=1.741\end{array}$ & $\begin{array}{l}\text { C3-C2-C1=123.526 } \\
\text { C4-C3-C2=116.339 } \\
\text { C5-C4-C3=121.157 } \\
\text { C6-C5-C4=121.419 } \\
\text { C7-C1-C6=136.095 } \\
\text { C8-C7-C1=105.171 } \\
\text { C9-C8-C7=122.640 } \\
\text { C10-C9-C8=117.609 } \\
\text { C11-C10-C9=120.861 } \\
\text { C12-C11-C10=121.222 } \\
\text { O13-C8-C7 =111.953 } \\
\text { Cl14-C3-C2=120.109 } \\
\text { Cl15-C4-C3=120.380 } \\
\text { H16-C5-C4=118.349 } \\
\text { H17-C6-C5=120.304 } \\
\text { Cl18-C9-C8=121.136 } \\
\text { H19-C10-C9=119.785 } \\
\text { Cl20-C11-C10=117.772 } \\
\text { Cl21-Cl20-C11=121.595 }\end{array}$ & $\begin{array}{l}\text { C4-C3-C2-C1=0.000 } \\
\text { C5-C4-C3-C2=0.000 } \\
\text { C6-C5-C4-C3 =0.000 } \\
\text { C7-C1-C6-C5=180.000 } \\
\text { C8-C7-C1-C6 }=180.000 \\
\text { C9-C8-C7-C1=180.000 } \\
\text { C10-C9-C8-C7=0.000 } \\
\text { C11-C10-C9-C8=0.000 } \\
\text { C12-C11-C10-C9=0.000 } \\
\text { O13-C8-C7-C1 =0.000 } \\
\text { Cl14-C3-C2-C1=180.000 } \\
\text { Cl15-C4-C3-C2=180.000 } \\
\text { H16-C5-C4-C3=180.000 } \\
\text { H17-C6-C5-C4=180.000 } \\
\text { Cl18-C9-C8-C7=180.000 } \\
\text { H19-C10-C9-C8=180.000 } \\
\text { Cl20-C11-C10-C9=180.000 } \\
\text { Cl21-Cl20-C11-C10 =180.000 }\end{array}$ \\
\hline 15 & $\begin{array}{l}\mathrm{C} 2-\mathrm{C} 1=1.407 \\
\mathrm{C} 3-\mathrm{C} 2=1.386 \\
\mathrm{C} 4-\mathrm{C} 3=1.394 \\
\mathrm{C} 5-\mathrm{C} 4=1.404 \\
\mathrm{C} 6-\mathrm{C} 5=1.392 \\
\mathrm{C} 7-\mathrm{C} 1=1.453 \\
\mathrm{C} 8-\mathrm{C} 7=1.406 \\
\mathrm{C} 9-\mathrm{C} 8=1.389 \\
\mathrm{C} 10-\mathrm{C} 9=1.403 \\
\mathrm{C} 11-\mathrm{C} 10=1.417 \\
\mathrm{C} 12-\mathrm{C} 11=1.399 \\
\mathrm{O} 13-\mathrm{C} 8=1.364 \\
\mathrm{H} 14-\mathrm{C} 3=1.084\end{array}$ & $\begin{array}{l}\text { C3-C2-C1=123.495 } \\
\text { C4-C3-C2=116.876 } \\
\text { C5-C4-C3=120.374 } \\
\text { C6-C5-C4=122.504 } \\
\text { C7-C1-C6 }=135.668 \\
\text { C8-C7-C1=105.246 } \\
\text { C9-C8-C7=123.367 } \\
\text { C10-C9-C8 }=117.317 \\
\text { C11-C10-C9 }=120.675 \\
\text { C12-C11-C10=136.017 } \\
\text { O13-C8-C7 }=112.057 \\
\text { H14-C3-C2 }=121.385 \\
\text { H15-C4-C3 }=120.188\end{array}$ & 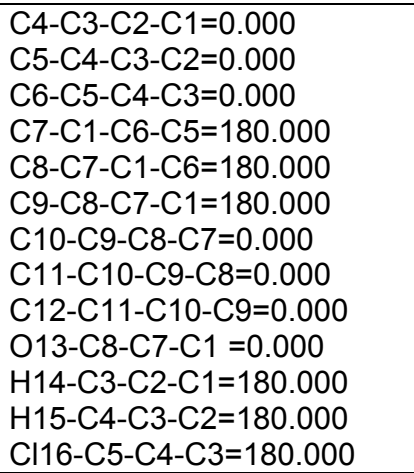 \\
\hline
\end{tabular}




\begin{tabular}{|c|c|c|c|}
\hline & $\begin{array}{l}\mathrm{H} 15-\mathrm{C} 4=1.084 \\
\mathrm{Cl} 16-\mathrm{C} 5=1.758 \\
\mathrm{H} 17-\mathrm{C} 6=1.082 \\
\mathrm{Cl} 18-\mathrm{C} 9=1.733 \\
\mathrm{Cl} 19-\mathrm{C} 10=1.735 \\
\mathrm{Cl} 20-\mathrm{C} 11=1.738 \\
\mathrm{Cl} 21-\mathrm{C} 12=1.741 \\
\end{array}$ & $\begin{array}{l}\mathrm{Cl} 16-\mathrm{C} 5-\mathrm{C} 4=118.628 \\
\mathrm{H} 17-\mathrm{C} 6-\mathrm{C} 5=120.760 \\
\mathrm{Cl} 18-\mathrm{C} 9-\mathrm{C} 8=119.638 \\
\mathrm{Cl} 19-\mathrm{C} 10-\mathrm{C} 9=119.249 \\
\mathrm{Cl} 20-\mathrm{C} 11-\mathrm{C} 10=119.714 \\
\mathrm{Cl} 21-\mathrm{Cl} 20-\mathrm{C} 11=119.431\end{array}$ & $\begin{array}{l}\mathrm{H} 17-\mathrm{C} 6-\mathrm{C} 5-\mathrm{C} 4=180.000 \\
\mathrm{Cl} 18-\mathrm{C} 9-\mathrm{C} 8-\mathrm{C} 7=180.000 \\
\mathrm{Cl} 19-\mathrm{C} 10-\mathrm{C} 9-\mathrm{C} 8=180.000 \\
\mathrm{Cl} 20-\mathrm{C} 11-\mathrm{C} 10-\mathrm{C} 9=180.000 \\
\mathrm{Cl} 21-\mathrm{Cl} 20-\mathrm{C} 11-\mathrm{C} 10=0.000\end{array}$ \\
\hline 16 & $\begin{array}{l}\mathrm{C} 2-\mathrm{C} 1=1.407 \\
\mathrm{C} 3-\mathrm{C} 2=1.384 \\
\mathrm{C} 4-\mathrm{C} 3=1.396 \\
\mathrm{C} 5-\mathrm{C} 4=1.412 \\
\mathrm{C} 6-\mathrm{C} 5=1.394 \\
\mathrm{C} 7-\mathrm{C} 1=1.452 \\
\mathrm{C} 8-\mathrm{C} 7=1.407 \\
\mathrm{C} 9-\mathrm{C} 8=1.383 \\
\mathrm{C} 10-\mathrm{C} 9=1.394 \\
\mathrm{C} 11-\mathrm{C} 10=1.414 \\
\mathrm{C} 12-\mathrm{C} 11=1.399 \\
\mathrm{O} 13-\mathrm{C} 8=1.371 \\
\mathrm{H} 14-\mathrm{C} 3=1.083 \\
\mathrm{Cl} 15-\mathrm{C} 4=1.744 \\
\mathrm{Cl} 16-\mathrm{C} 5=1.748 \\
\mathrm{H} 17-\mathrm{C} 6=1.082 \\
\mathrm{H} 18-\mathrm{C} 9=1.083 \\
\mathrm{Cl} 19-\mathrm{C} 10=1.744 \\
\mathrm{Cl} 20-\mathrm{C} 11=1.738 \\
\mathrm{Cl} 21-\mathrm{C} 12=1.742\end{array}$ & $\begin{array}{l}\text { C3-C2-C1=123.507 } \\
\text { C4-C3-C2=116.983 } \\
\text { C5-C4-C3=120.870 } \\
\text { C6-C5-C4=120.976 } \\
\text { C7-C1-C6=136.121 } \\
\text { C8-C7-C1=105.437 } \\
\text { C9-C8-C7=123.798 } \\
\text { C10-C9-C8=116.804 } \\
\text { C11-C10-C9=121.778 } \\
\text { C12-C11-C10=136.242 } \\
\text { O13-C8-C7 =111.720 } \\
\text { H14-C3-C2=121.885 } \\
\text { Cl15-C4-C3=118.002 } \\
\text { Cl16-C5-C4=120.844 } \\
\text { H17-C6-C5=119.667 } \\
\text { H18-C9-C8=121.972 } \\
\text { Cl19-C10-C9=117.637 } \\
\text { Cl20-C11-C10=120.253 } \\
\text { Cl21-CI20-C11=119.286 }\end{array}$ & 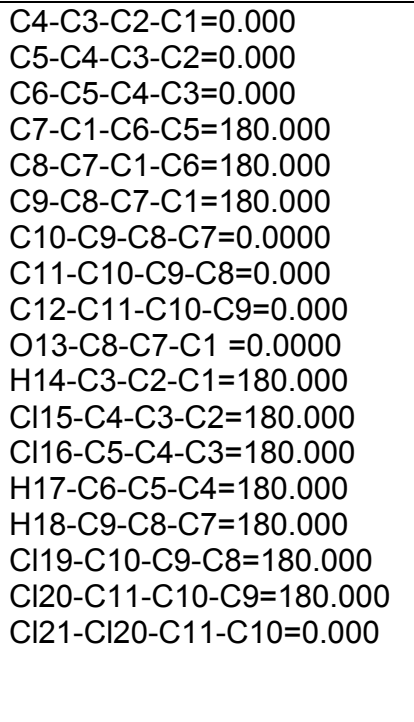 \\
\hline 17 & $\begin{array}{l}\mathrm{C} 2-\mathrm{C} 1=1.405 \\
\mathrm{C} 3-\mathrm{C} 2=1.384 \\
\mathrm{C} 4-\mathrm{C} 3=1.397 \\
\mathrm{C} 5-\mathrm{C} 4=1.413 \\
\mathrm{C} 6-\mathrm{C} 5=1.394 \\
\mathrm{C} 7-\mathrm{C} 1=1.451 \\
\mathrm{C} 8-\mathrm{C} 7=1.4054 \\
\mathrm{C} 9-\mathrm{C} 8=1.390 \\
\mathrm{C} 10-\mathrm{C} 9=1.406 \\
\mathrm{C} 11-\mathrm{C} 10=1.415 \\
\mathrm{C} 12-\mathrm{C} 11=1.392 \\
\mathrm{O} 13-\mathrm{C} 8=1.369 \\
\mathrm{H} 14-\mathrm{C} 3=1.083 \\
\mathrm{Cl} 15-\mathrm{C} 4=1.744 \\
\mathrm{Cl} 16-\mathrm{C} 5=1.747 \\
\mathrm{H} 17-\mathrm{C} 6=1.084 \\
\mathrm{Cl} 18-\mathrm{C} 9=1.734 \\
\mathrm{Cl} 19-\mathrm{C} 10=1.735 \\
\mathrm{Cl} 20-\mathrm{C} 11=1.748 \\
\mathrm{H} 21-\mathrm{C} 12=1.084\end{array}$ & $\begin{array}{l}\text { C3-C2-C1=123.379 } \\
\text { C4-C3-C2=116.916 } \\
\text { C5-C4-C3=120.990 } \\
\text { C6-C5-C4=120.828 } \\
\text { C7-C1-C6=135.566 } \\
\text { C8-C7-C1=105.039 } \\
\text { C9-C8-C7=122.826 } \\
\text { C10-C9-C8=117.535 } \\
\text { C11-C10-C9=119.872 } \\
\text { C12-C11-C10=121.677 } \\
\text { O13-C8-C7 =112.178 } \\
\text { H14-C3-C2=121.978 } \\
\text { Cl15-C4-C3=117.996 } \\
\text { Cl16-C5-C4=120.909 } \\
\text { H17-C6-C5=119.496 } \\
\text { Cl18-C9-C8=119.625 } \\
\text { Cl19-C10-C9=119.589 } \\
\text { Cl20-C11-C10=120.385 } \\
\text { H21-Cl20-C11=119.635 }\end{array}$ & $\begin{array}{l}\text { C4-C3-C2-C1=0.000 } \\
\text { C5-C4-C3-C2=0.000 } \\
\text { C6-C5-C4-C3=0.000 } \\
\text { C7-C1-C6-C5=180.000 } \\
\text { C8-C7-C1-C6=180.000 } \\
\text { C9-C8-C7-C1=180.000 } \\
\text { C10-C9-C8-C7=0.000 } \\
\text { C11-C10-C9-C8=0.000 } \\
\text { C12-C11-C10-C9=0.000 } \\
\text { O13-C8-C7-C1 =0.000 } \\
\text { H14-C3-C2-C1=180.000 } \\
\text { Cl15-C4-C3-C2=180.000 } \\
\text { Cl16-C5-C4-C3=180.000 } \\
\text { H17-C6-C5-C4=180.000 } \\
\text { Cl18-C9-C8-C7=180.000 } \\
\text { Cl19-C10-C9-C8=180.000 } \\
\text { Cl20-C11-C10-C9=180.000 } \\
\text { H21-Cl20-C11-C10=180.000 }\end{array}$ \\
\hline 18 & 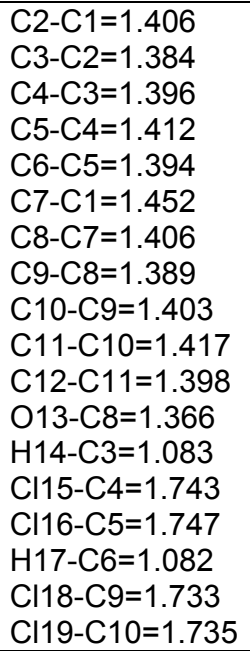 & $\begin{array}{l}\text { C3-C2-C1=123.624 } \\
\text { C4-C3-C2=116.882 } \\
\text { C5-C4-C3=120.886 } \\
\text { C6-C5-C4=121.000 } \\
\text { C7-C1-C6=136.067 } \\
\text { C8-C7-C1=105.195 } \\
\text { C9-C8-C7=123.391 } \\
\text { C10-C9-C8=117.273 } \\
\text { C11-C10-C9=120.696 } \\
\text { C12-C11-C10=136.029 } \\
\text { O13-C8-C7 =112.050 } \\
\text { H14-C3-C2=121.914 } \\
\text { Cl15-C4-C3=118.028 } \\
\text { Cl16-C5-C4=120.844 } \\
\text { H17-C6-C5=119.674 } \\
\text { Cl18-C9-C8=119.660 } \\
\text { Cl19-C10-C9=119.221 } \\
\text { Cl20-C11-C10 }=119.732\end{array}$ & $\begin{array}{l}\text { C4-C3-C2-C1 }=0.000 \\
\text { C5-C4-C3-C2 }=0.000 \\
\text { C6-C5-C4-C3 }=0.000 \\
\text { C7-C1-C6-C5 }=180.000 \\
\text { C8-C7-C1-C6 }=180.000 \\
\text { C9-C8-C7-C1 }=180.000 \\
\text { C10-C9-C8-C7 }=0.000 \\
\text { C11-C10-C9-C8 }=0.000 \\
\text { C12-C11-C10-C9=0.000 } \\
\text { O13-C8-C7-C1 }=0.000 \\
\text { H14-C3-C2-C1 } 180.000 \\
\text { Cl15-C4-C3-C2 }=180.000 \\
\text { Cl16-C5-C4-C3=180.000 } \\
\text { H17-C6-C5-C4=180.000 } \\
\text { Cl18-C9-C8-C7=180.000 } \\
\text { Cl19-C10-C9-C8 }=180.000 \\
\text { Cl20-C11-C10-C9=180.000 } \\
\text { Cl21-Cl20-C11-C } 10=0.000\end{array}$ \\
\hline
\end{tabular}




\begin{tabular}{|c|c|c|c|}
\hline & $\begin{array}{l}\mathrm{Cl} 20-\mathrm{C} 11=1.738 \\
\mathrm{Cl} 21-\mathrm{C} 12=1.740\end{array}$ & $\mathrm{Cl} 21-\mathrm{Cl} 20-\mathrm{C} 11=119.384$ & \\
\hline 19 & 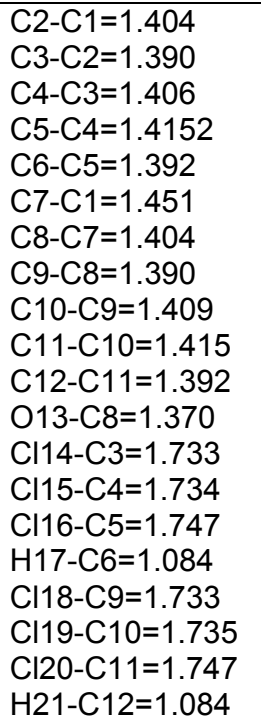 & $\begin{array}{l}\text { C3-C2-C1=122.932 } \\
\text { C4-C3-C2=117.402 } \\
\text { C5-C4-C3=119.918 } \\
\text { C6-C5-C4=121.701 } \\
\text { C7-C1-C6=135.335 } \\
\text { C8-C7-C1=105.107 } \\
\text { C9-C8-C7=122.931 } \\
\text { C10-C9-C8=117.394 } \\
\text { C11-C10-C9=119.932 } \\
\text { C12-C11-C10=121.693 } \\
\text { O13-C8-C7 =112.091 } \\
\text { Cl14-C3-C2=119.716 } \\
\text { Cl15-C4-C3=119.587 } \\
\text { Cl16-C5-C4=120.374 } \\
\mathrm{H} 17-C 6-C 5=119.8 \\
\text { Cl18-C9-C8=119.703 } \\
\text { Cl19-C10-C9=119.566 } \\
\text { Cl20-C11-C10=120.363 } \\
\mathrm{H} 21-C \mid 20-C 11=119.681\end{array}$ & $\begin{array}{l}\text { C4-C3-C2-C1=0.000 } \\
\text { C5-C4-C3-C2=0.000 } \\
\text { C6-C5-C4-C3=0.000 } \\
\text { C7-C1-C6-C5=180.000 } \\
\text { C8-C7-C1-C6=180.000 } \\
\text { C9-C8-C7-C1=180.000 } \\
\text { C10-C9-C8-C7=0.000 } \\
\text { C11-C10-C9-C8=0.000 } \\
\text { C12-C11-C10-C9=0.000 } \\
\text { O13-C8-C7-C1 =0.000 } \\
\text { Cl14-C3-C2-C1=180.000 } \\
\text { Cl15-C4-C3-C2=180.000 } \\
\text { Cl16-C5-C4-C3=180.000 } \\
\text { H17-C6-C5-C4=180.000 } \\
\text { Cl18-C9-C8-C7=180.000 } \\
\text { Cl19-C10-C9-C8=180.000 } \\
\text { Cl20-C11-C10-C9=180.000 } \\
\text { H21-Cl20-C11-C10=180.000 }\end{array}$ \\
\hline 20 & $\begin{array}{l}\mathrm{C} 2-\mathrm{C} 1=1.408 \\
\mathrm{C} 3-\mathrm{C} 2=1.389 \\
\mathrm{C} 4-\mathrm{C} 3=1.394 \\
\mathrm{C} 5-\mathrm{C} 4=1.403 \\
\mathrm{C} 6-\mathrm{C} 5=1.392 \\
\mathrm{C} 7-\mathrm{C} 1=1.452 \\
\mathrm{C} 8-\mathrm{C} 7=1.405 \\
\mathrm{C} 9-\mathrm{C} 8=1.384 \\
\mathrm{C} 10-\mathrm{C} 9=1.396 \\
\mathrm{C} 11-\mathrm{C} 10=1.413 \\
\mathrm{C} 12-\mathrm{C} 11=1.394 \\
\mathrm{O} 13-\mathrm{C} 8=1.370 \\
\mathrm{Cl} 14-\mathrm{C} 3=1.743 \\
\mathrm{H} 15-\mathrm{C} 4=1.083 \\
\mathrm{Cl} 16-\mathrm{C} 5=1.757 \\
\mathrm{H} 17-\mathrm{C} 6=1.084 \\
\mathrm{H} 18-\mathrm{C} 9=1.083 \\
\mathrm{Cl} 19-\mathrm{C} 10=1.745 \\
\mathrm{Cl} 20-\mathrm{C} 11=1.748 \\
\mathrm{H} 21-\mathrm{C} 12=1.0844\end{array}$ & $\begin{array}{l}\text { C3-C2-C1=121.965 } \\
\text { C4-C3-C2=118.039 } \\
\text { C5-C4-C3=119.880 } \\
\text { C6-C5-C4=122.511 } \\
\text { C7-C1-C6=135.011 } \\
\text { C8-C7-C1=105.482 } \\
\text { C9-C8-C7=123.348 } \\
\text { C10-C9-C8=116.939 } \\
\text { C11-C10-C9=120.992 } \\
\text { C12-C11-C10=120.812 } \\
\text { O13-C8-C7 =112.199 } \\
\text { Cl14-C3-C2=120.924 } \\
\text { H15-C4-C3=A119.842 } \\
\text { Cl16-C5-C4=118.354 } \\
\text { H17-C6-C5=120.646 } \\
\text { H18-C9-C8=121.946 } \\
\text { Cl19-C10-C9=117.994 } \\
\text { Cl20-C11-C10=120.907 } \\
\text { H21-Cl20-C11=119.494 }\end{array}$ & $\begin{array}{l}\text { C4-C3-C2-C1=0.000 } \\
\text { C5-C4-C3-C2 =0.000 } \\
\text { C6-C5-C4-C3=0.000 } \\
\text { C7-C1-C6-C5=180.000 } \\
\text { C8-C7-C1-C6=180.000 } \\
\text { C9-C8-C7-C1=180.000 } \\
\text { C10-C9-C8-C7=0.000 } \\
\text { C11-C10-C9-C8=0.000 } \\
\text { C12-C11-C10-C9=0.000 } \\
\text { O13-C8-C7-C1 =180.000 } \\
\text { Cl14-C3-C2-C1=180.000 } \\
\text { H15-C4-C3-C2=180.000 } \\
\text { Cl16-C5-C4-C3=180.000 } \\
\text { H17-C6-C5-C4=180.000 } \\
\text { H18-C9-C8-C7=180.000 } \\
\text { Cl19-C10-C9-C8=180.000 } \\
\text { Cl20-C11-C10-C9=180.000 } \\
\text { H21-Cl20-C11-C10=180.000 }\end{array}$ \\
\hline 21 & $\begin{array}{l}\mathrm{C} 2-\mathrm{C} 1=1.410 \\
\mathrm{C} 3-\mathrm{C} 2=1.389 \\
\mathrm{C} 4-\mathrm{C} 3=1.394 \\
\mathrm{C} 5-\mathrm{C} 4=1.403 \\
\mathrm{C} 6-\mathrm{C} 5=1.393 \\
\mathrm{C} 7-\mathrm{C} 1=1.453 \\
\mathrm{C} 8-\mathrm{C} 7=1.406 \\
\mathrm{C} 9-\mathrm{C} 8=1.383 \\
\mathrm{C} 10-\mathrm{C} 9=1.394 \\
\mathrm{C} 11-\mathrm{C} 10=1.414 \\
\mathrm{C} 12-\mathrm{C} 11=\mathrm{B} 1.399 \\
\mathrm{O} 13-\mathrm{C} 8=1.370 \\
\mathrm{Cl} 14-\mathrm{C} 3=1.746 \\
\mathrm{H} 15-\mathrm{C} 4=1.085 \\
\mathrm{C} 116-\mathrm{C} 5=1.086 \\
\mathrm{H} 17-\mathrm{C} 6=1.083 \\
\mathrm{H} 18-\mathrm{C} 9=1.083 \\
\mathrm{Cl} 19-\mathrm{C} 10=1.745 \\
\mathrm{Cl} 20-\mathrm{C} 11=1.739 \\
\mathrm{H} 21-\mathrm{C} 12=1.743\end{array}$ & $\begin{array}{l}\text { C3-C2-C1=122.449 } \\
\text { C4-C3-C2=117.667 } \\
\text { C5-C4-C3=120.544 } \\
\text { C6-C5-C4=121.662 } \\
\text { C7-C1-C6=136.011 } \\
\text { C8-C7-C1=105.663 } \\
\text { C9-C8-C7=123.937 } \\
\text { C10-C9-C8=116.758 } \\
\text { C11-C10-C9=121.745 } \\
\text { C12-C11-C10=136.146 } \\
\text { O13-C8-C7 =111.670 } \\
\text { Cl14-C3-C2=120.763 } \\
\text { H15-C4-C3=119.033 } \\
\text { Cl16-C5-C4=118.772 } \\
\text { H17-C6-C5=120.852 } \\
\text { H18-C9-C8=121.948 } \\
\text { Cl19-C10-C9=117.680 } \\
\text { Cl20-C11-C10=120.193 } \\
\text { H21-Cl20-C11=119.376 }\end{array}$ & 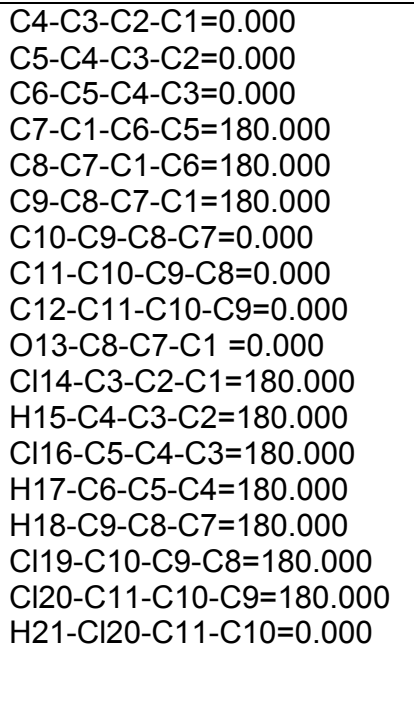 \\
\hline 22 & $\begin{array}{l}C 2-C 1=1.408 \\
C 3-C 2=1.386 \\
C 4-C 3=1.394\end{array}$ & $\begin{array}{l}\text { C3-C2-C1=123.946 } \\
C 4-C 3-C 2=115.756 \\
C 5-C 4-C 3=122.390\end{array}$ & $\begin{array}{l}\text { C4-C3-C2-C1 }=0.000 \\
\text { C5-C4-C3-C2 }=0.000 \\
\text { C6-C5-C4-C3 }=0.000\end{array}$ \\
\hline
\end{tabular}




\begin{tabular}{|c|c|c|c|}
\hline & $\begin{array}{l}\mathrm{C} 5-\mathrm{C} 4=1.403 \\
\mathrm{C} 6-\mathrm{C} 5=1.392 \\
\mathrm{C} 7-\mathrm{C} 1=1.452 \\
\mathrm{C} 8-\mathrm{C} 7=1.407 \\
\mathrm{C} 9-\mathrm{C} 8=1.383 \\
\mathrm{C} 10-\mathrm{C} 9=1.394 \\
\mathrm{C} 11-\mathrm{C} 10=1.413 \\
\mathrm{C} 12-\mathrm{C} 11=1.399 \\
\mathrm{O} 13-\mathrm{C} 8=1.371 \\
\mathrm{Cl} 14-\mathrm{C} 3=1.083 \\
\mathrm{H} 15-\mathrm{C} 4=1.755 \\
\mathrm{Cl} 16-\mathrm{C} 5=1.084 \\
\mathrm{H} 17-\mathrm{C} 6=1.083 \\
\mathrm{H} 18-\mathrm{C} 9=1.083 \\
\mathrm{Cl} 19-\mathrm{C} 10=1.745 \\
\mathrm{Cl} 20-\mathrm{C} 11=1.739 \\
\mathrm{H} 21-\mathrm{C} 12=1.743\end{array}$ & $\begin{array}{l}\text { C6-C5-C4=120.356 } \\
\text { C7-C1-C6=136.410 } \\
\text { C8-C7-C1=105.536 } \\
\text { C9-C8-C7=123.849 } \\
\text { C10-C9-C8=116.823 } \\
\text { C11-C10-C9=121.740 } \\
\text { C12-C11-C10=136.256 } \\
\text { O13-C8-C7 =111.662 } \\
\text { Cl14-C3-C2=122.019 } \\
\text { H15-C4-C3=118.692 } \\
\text { Cl16-C5-C4=119.330 } \\
\text { H17-C6-C5=120.123 } \\
\text { H18-C9-C8=121.952 } \\
\text { Cl19-C10-C9=117.652 } \\
\text { Cl20-C11-C10=120.239 } \\
\text { H21-Cl20-C11=119.281 }\end{array}$ & 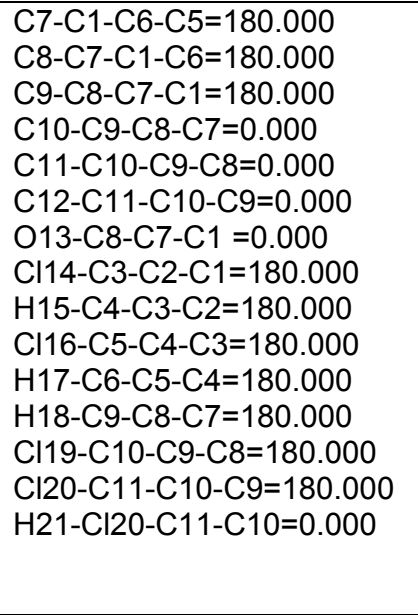 \\
\hline 23 & $\begin{array}{l}\mathrm{C} 2-\mathrm{C} 1=1.406 \\
\mathrm{C} 3-\mathrm{C} 2=1.383 \\
\mathrm{C} 4-\mathrm{C} 3=1.396 \\
\mathrm{C} 5-\mathrm{C} 4=1.412 \\
\mathrm{C} 6-\mathrm{C} 5=1.394 \\
\mathrm{C} 7-\mathrm{C} 1=1.450 \\
\mathrm{C} 8-\mathrm{C} 7=1.409 \\
\mathrm{C} 9-\mathrm{C} 8=1.392 \\
\mathrm{C} 10-\mathrm{C} 9=1.402 \\
\mathrm{C} 11-\mathrm{C} 10=1.403 \\
\mathrm{C} 12-\mathrm{C} 11=1.390 \\
\mathrm{O} 13-\mathrm{C} 8=1.367 \\
\mathrm{Cl} 14-\mathrm{C} 3=1.083 \\
\mathrm{H} 15-\mathrm{C} 4=1.744 \\
\mathrm{Cl} 16-\mathrm{C} 5=1.747 \\
\mathrm{H} 17-\mathrm{C} 6=1.083 \\
\mathrm{H} 18-\mathrm{C} 9=1.733 \\
\mathrm{Cl} 19-\mathrm{C} 10=1.743 \\
\mathrm{Cl} 20-\mathrm{C} 11=1.083 \\
\mathrm{H} 21-\mathrm{C} 12=1.749\end{array}$ & $\begin{array}{l}\text { C3-C2-C1=123.561 } \\
\text { C4-C3-C2=116.888 } \\
\text { C5-C4-C3=120.908 } \\
\text { C6-C5-C4=120.989 } \\
\text { C7-C1-C6=135.922 } \\
\text { C8-C7-C1=105.279 } \\
\text { C9-C8-C7=123.842 } \\
\text { C10-C9-C8=116.374 } \\
\text { C11-C10-C9=121.412 } \\
\text { C12-C11-C10=120.512 } \\
\text { O13-C8-C7 =111.812 } \\
\text { Cl14-C3-C2=121.935 } \\
\text { H15-C4-C3=117.989 } \\
\text { Cl16-C5-C4=120.858 } \\
\text { H17-C6-C5=119.705 } \\
\text { H18-C9-C8=120.184 } \\
\text { Cl19-C10-C9=120.505 } \\
\text { Cl20-C11-C10=119.299 } \\
\text { H21-Cl20-C11=119.392 }\end{array}$ & 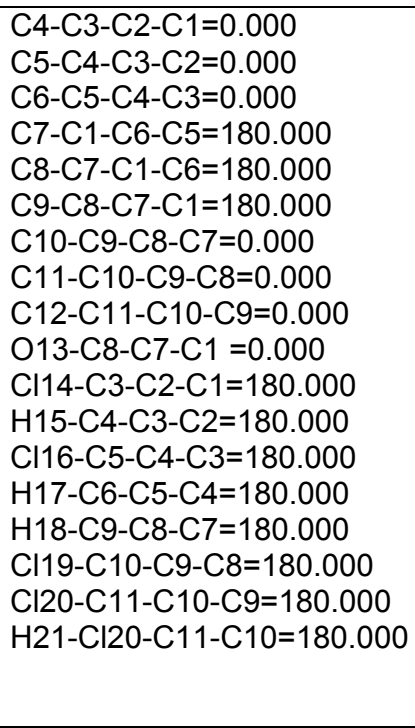 \\
\hline 24 & $\begin{array}{l}\mathrm{C} 2-\mathrm{C} 1=1.410 \\
\mathrm{C} 3-\mathrm{C} 2=1.385 \\
\mathrm{C} 4-\mathrm{C} 3=1.395 \\
\mathrm{C} 5-\mathrm{C} 4=1.402 \\
\mathrm{C} 6-\mathrm{C} 5=1.392 \\
\mathrm{C} 7-\mathrm{C} 1=1.450 \\
\mathrm{C} 8-\mathrm{C} 7=1.405 \\
\mathrm{C} 9-\mathrm{C} 8=1.389 \\
\mathrm{C} 10-\mathrm{C} 9=1.405 \\
\mathrm{C} 11-\mathrm{C} 10=1.414 \\
\mathrm{C} 12-\mathrm{C} 11=1.392 \\
\mathrm{O} 13-\mathrm{C} 8=1.369 \\
\mathrm{Cl} 14-\mathrm{C} 3=1.083 \\
\mathrm{H} 15-\mathrm{C} 4=1.752 \\
\mathrm{Cl} 16-\mathrm{C} 5=1.083 \\
\mathrm{H} 17-\mathrm{C} 6=1.750 \\
\mathrm{H} 18-\mathrm{C} 9=1.734 \\
\mathrm{Cl} 19-\mathrm{C} 10=1.735 \\
\mathrm{Cl} 20-\mathrm{C} 11=1.747 \\
\mathrm{H} 21-\mathrm{C} 12=1.0822\end{array}$ & $\begin{array}{l}\text { C3-C2-C1=124.421 } \\
\text { C4-C3-C2=115.657 } \\
\text { C5-C4-C3=122.619 } \\
\text { C6-C5-C4=119.538 } \\
\text { C7-C1-C6=136.910 } \\
\text { C8-C7-C1=104.892 } \\
\text { C9-C8-C7=123.028 } \\
\text { C10-C9-C8=117.495 } \\
\text { C11-C10-C9=119.797 } \\
\text { C12-C11-C10=121.826 } \\
\text { O13-C8-C7 =112.186 } \\
\text { Cl14-C3-C2=122.097 } \\
\text { H15-C4-C3=118.898 } \\
\text { Cl16-C5-C4=120.278 } \\
\text { H17-C6-C5=119.254 } \\
\text { H18-C9-C8=119.624 } \\
\text { Cl19-C10-C9=119.587 } \\
\text { Cl20-C11-C10 =120.327 } \\
\text { H21-Cl20-C11=119.854 }\end{array}$ & 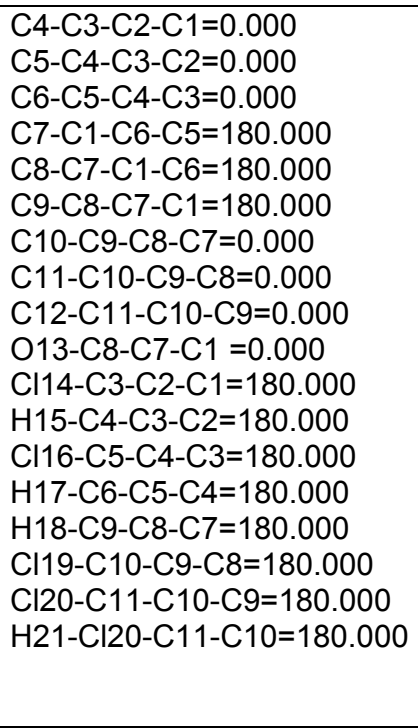 \\
\hline 25 & $\begin{array}{l}C 2-C 1=1.418 \\
C 3-C 2=1.385 \\
C 4-C 3=1.388 \\
C 5-C 4=1.398 \\
\text { C6-C5 }=1.395 \\
\text { C7-C1 }=1.473 \\
\text { C8-C7 }=1.412 \\
\text { C9-C8 }=1.382\end{array}$ & $\begin{array}{l}\text { C3-C2-C1 }=126.368 \\
\text { C4-C3-C2 }=115.781 \\
\text { C5-C4-C3 }=121.477 \\
\text { C6-C5-C4 }=120.900 \\
\text { C7-C1-C6 }=140.587 \\
\text { C8-C7-C1 }=104.668 \\
\text { C9-C8-C7 }=125.618 \\
\text { C10-C9-C8 }=116.898\end{array}$ & $\begin{array}{l}\text { C4-C3-C2-C1 }=0.000 \\
\text { C5-C4-C3-C2 }=0.000 \\
\text { C6-C5-C4-C3 }=0.000 \\
\text { C7-C1-C6-C5 }=180.000 \\
\text { C8-C7-C1-C6 }=180.000 \\
\text { C9-C8-C7-C1 }=180.000 \\
\text { C10-C9-C8-C7 }=0.000 \\
\text { C11-C10-C9-C8 }=0.000\end{array}$ \\
\hline
\end{tabular}




\begin{tabular}{|c|c|c|c|}
\hline & $\begin{array}{l}\mathrm{C} 10-\mathrm{C} 9=1.388 \\
\mathrm{C} 11-\mathrm{C} 10=1.410 \\
\mathrm{C} 12-\mathrm{C} 11=1.409 \\
\mathrm{O} 13-\mathrm{C} 8=1.364 \\
\mathrm{Cl} 14-\mathrm{C} 3=1.083 \\
\mathrm{H} 15-\mathrm{C} 4=1.750 \\
\mathrm{Cl} 16-\mathrm{C} 5=1.083 \\
\mathrm{H} 17-\mathrm{C} 6=1.747 \\
\mathrm{H} 18-\mathrm{C} 9=1.083 \\
\mathrm{Cl} 19-\mathrm{C} 10=1.743 \\
\mathrm{Cl} 20-\mathrm{C} 11=1.740 \\
\mathrm{H} 21-\mathrm{C} 12=1.735\end{array}$ & $\begin{array}{l}\mathrm{C} 11-\mathrm{C} 10-\mathrm{C} 9=120.868 \\
\mathrm{C} 12-\mathrm{C} 11-\mathrm{C} 10=120.563 \\
\mathrm{O} 13-\mathrm{C} 8-\mathrm{C} 7=112.341 \\
\mathrm{Cl} 14-\mathrm{C} 3-\mathrm{C} 2=121.393 \\
\mathrm{H} 15-\mathrm{C} 4-\mathrm{C} 3=119.508 \\
\mathrm{Cl} 16-\mathrm{C} 5-\mathrm{C} 4=119.880 \\
\mathrm{H} 17-\mathrm{C} 6-\mathrm{C} 5=114.931 \\
\mathrm{H} 18-\mathrm{C} 9-\mathrm{C} 8=121.367 \\
\mathrm{Cl} 19-\mathrm{C} 10-\mathrm{C} 9=117.914 \\
\mathrm{Cl} 20-\mathrm{C} 11-\mathrm{C} 10=119.202 \\
\mathrm{H} 21-\mathrm{Cl} 20-\mathrm{C} 11=118.021\end{array}$ & $\begin{array}{l}\mathrm{C} 12-\mathrm{C} 11-\mathrm{C} 10-\mathrm{C} 9=0.000 \\
\mathrm{O} 13-\mathrm{C} 8-\mathrm{C} 7-\mathrm{C} 1=0.000 \\
\mathrm{Cl} 14-\mathrm{C} 3-\mathrm{C} 2-\mathrm{C} 1=180.000 \\
\mathrm{H} 15-\mathrm{C} 4-\mathrm{C} 3-\mathrm{C} 2=180.000 \\
\mathrm{Cl} 16-\mathrm{C} 5-\mathrm{C} 4-\mathrm{C} 3=180.000 \\
\mathrm{H} 17-\mathrm{C} 6-\mathrm{C} 5-\mathrm{C} 4=180.000 \\
\mathrm{H} 18-\mathrm{C} 9-\mathrm{C} 8-\mathrm{C} 7=180.000 \\
\mathrm{Cl} 19-\mathrm{C} 10-\mathrm{C} 9-\mathrm{C} 8=180.000 \\
\mathrm{Cl} 20-\mathrm{C} 11-\mathrm{C} 10-\mathrm{C} 9=180.000 \\
\mathrm{H} 21-\mathrm{Cl} 20-\mathrm{C} 11-\mathrm{C} 10=180.000\end{array}$ \\
\hline 26 & $\begin{array}{l}\mathrm{C} 2-\mathrm{C} 1=1.406 \\
\mathrm{C} 3-\mathrm{C} 2=1.387 \\
\mathrm{C} 4-\mathrm{C} 3=1.394 \\
\mathrm{C} 5-\mathrm{C} 4=1.405 \\
\mathrm{C} 6-\mathrm{C} 5=1.392 \\
\mathrm{C} 7-\mathrm{C} 1=1.451 \\
\mathrm{C} 8-\mathrm{C} 7=1.405 \\
\mathrm{C} 9-\mathrm{C} 8=1.310 \\
\mathrm{C} 10-\mathrm{C} 9=1.406 \\
\mathrm{C} 11-\mathrm{C} 10=1.414 \\
\mathrm{C} 12-\mathrm{C} 11=1.392 \\
\mathrm{O} 13-\mathrm{C} 8=1.369 \\
\mathrm{Cl} 14-\mathrm{C} 3=1.083 \\
\mathrm{Cl} 15-\mathrm{C} 4=1.755 \\
\mathrm{H} 16-\mathrm{C} 5=1.084 \\
\mathrm{H} 17-\mathrm{C} 6=1.086 \\
\mathrm{Cl} 18-\mathrm{C} 9=1.735 \\
\mathrm{Cl} 19-\mathrm{C} 10=1.736 \\
\mathrm{Cl} 20-\mathrm{C} 11=1.748 \\
\mathrm{H} 21-\mathrm{C} 12=1.084\end{array}$ & $\begin{array}{l}\text { C3-C2-C1=123.806 } \\
\text { C4-C3-C2=115.668 } \\
\text { C5-C4-C3=122.542 } \\
\text { C6-C5-C4=120.189 } \\
\text { C7-C1-C6=135.810 } \\
\text { C8-C7-C1=105.151 } \\
\text { C9-C8-C7=122.851 } \\
\text { C10-C9-C8=117.583 } \\
\text { C11-C10-C9=119.809 } \\
\text { C12-C11-C10=121.703 } \\
\text { O13-C8-C7 =112.101 } \\
\text { Cl14-C3-C2=122.119 } \\
\text { CL15-C4-C3=118.667 } \\
\text { H16-C5-C4=119.290 } \\
\text { H17-C6-C5=120.040 } \\
\text { Cl18-C9-C8=119.629 } \\
\text { Cl19-C10-C9=119.631 } \\
\text { Cl20-C11-C10=120.384 } \\
\text { H21-Cl20-C11=119.596 }\end{array}$ & 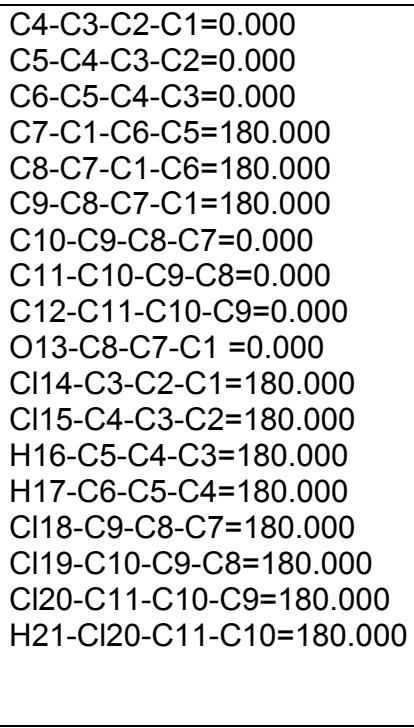 \\
\hline 27 & $\begin{array}{l}\mathrm{C} 2-\mathrm{C} 1=1.408 \\
\mathrm{C} 3-\mathrm{C} 2=1.386 \\
\mathrm{C} 4-\mathrm{C} 3=1.394 \\
\mathrm{C} 5-\mathrm{C} 4=1.403 \\
\mathrm{C} 6-\mathrm{C} 5=1.392 \\
\mathrm{C} 7-\mathrm{C} 1=1.452 \\
\mathrm{C} 8-\mathrm{C} 7=1.407 \\
\mathrm{C} 9-\mathrm{C} 8=1.383 \\
\mathrm{C} 10-\mathrm{C} 9=1.394 \\
\mathrm{C} 11-\mathrm{C} 10=1.413 \\
\mathrm{C} 12-\mathrm{C} 11=1.399 \\
\mathrm{O} 13-\mathrm{C} 8=1.371 \\
\mathrm{H} 14-\mathrm{C} 3=1.083 \\
\mathrm{Cl} 15-\mathrm{C} 4=1.755 \\
\mathrm{H} 16-\mathrm{C} 5=1.084 \\
\mathrm{H} 17-\mathrm{C} 6=1.083 \\
\mathrm{H} 18-\mathrm{C} 9=1.083 \\
\mathrm{Cl} 19-\mathrm{C} 10=1.745 \\
\mathrm{Cl} 20-\mathrm{C} 11=1.738 \\
\mathrm{Cl} 21-\mathrm{C} 12=1.743\end{array}$ & $\begin{array}{l}\text { C3-C2-C1=123.929 } \\
\text { C4-C3-C2=115.766 } \\
\text { C5-C4-C3=122.388 } \\
\text { C6-C5-C4=120.357 } \\
\text { C7-C1-C6=136.392 } \\
\text { C8-C7-C1=105.532 } \\
\text { C9-C8-C7=123.856 } \\
\text { C10-C9-C8=116.825 } \\
\text { C11-C10-C9=121.740 } \\
\text { C12-C11-C10=136.280 } \\
\text { O13-C8-C7 =111.666 } \\
\text { H14-C3-C2=122.002 } \\
\text { CI15-C4-C3=118.696 } \\
\text { H16-C5-C4=119.330 } \\
\text { H17-C6-C5=120.154 } \\
\text { H18-C9-C8=121.961 } \\
\text { CI19-C10-C9=117.653 } \\
\text { Cl20-C11-C10=120.231 } \\
\text { Cl21-Cl20-C11=119.254 }\end{array}$ & 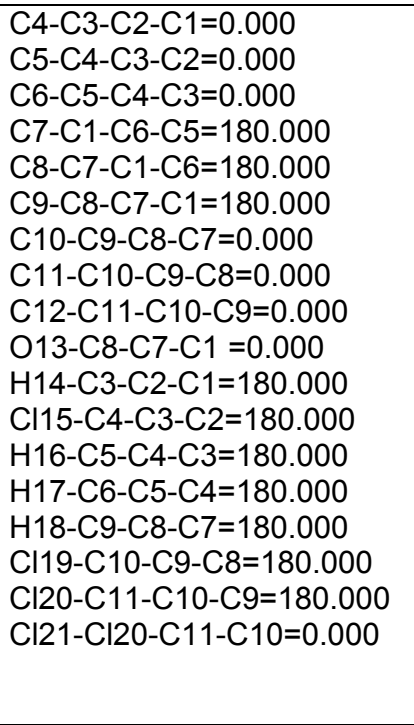 \\
\hline 28 & $\begin{array}{l}\mathrm{C} 2-\mathrm{C} 1=1.406 \\
\mathrm{C} 3-\mathrm{C} 2=1.383 \\
\mathrm{C} 4-\mathrm{C} 3=1.396 \\
\mathrm{C} 5-\mathrm{C} 4=1.412 \\
\mathrm{C} 6-\mathrm{C} 5=1.394 \\
\mathrm{C} 7-\mathrm{C} 1=1.450 \\
\mathrm{C} 8-\mathrm{C} 7=1.409 \\
\mathrm{C} 9-\mathrm{C} 8=1.392 \\
\mathrm{C} 10-\mathrm{C} 9=1.402 \\
\mathrm{C} 11-\mathrm{C} 10=1.403 \\
\mathrm{C} 12-\mathrm{C} 11=1.390 \\
\mathrm{O} 13-\mathrm{C} 8=1.367 \\
\mathrm{H} 14-\mathrm{C} 3=1.083\end{array}$ & $\begin{array}{l}\text { C3-C2-C1=123.561 } \\
\text { C4-C3-C2=116.888 } \\
\text { C5-C4-C3=120.908 } \\
\text { C6-C5-C4=120.989 } \\
\text { C7-C1-C6 }=135.922 \\
\text { C8-C7-C1=105.279 } \\
\text { C9-C8-C7=123.842 } \\
\text { C10-C9-C8 }=116.374 \\
\text { C11-C10-C9 }=121.412 \\
\text { C12-C11-C10 }=120.512 \\
\text { O13-C8-C7 }=111.812 \\
\text { H14-C3-C2 }=121.935 \\
\text { Cl15-C4-C3 }=117.989\end{array}$ & $\begin{array}{l}\text { C4-C3-C2-C1 }=0.000 \\
\text { C5-C4-C3-C2 }=0.000 \\
\text { C6-C5-C4-C3=0.000 } \\
\text { C7-C1-C6-C5 }=180.000 \\
\text { C8-C7-C1-C6 }=180.000 \\
\text { C9-C8-C7-C1 }=180.000 \\
\text { C10-C9-C8-C7 }=0.000 \\
\text { C11-C10-C9-C8 }=0.000 \\
\text { C12-C11-C10-C9 }=0.000 \\
\text { O13-C8-C7-C1 }=0.000 \\
\text { H14-C3-C2-C1=180.000 } \\
\text { Cl15-C4-C3-C2 }=180.000 \\
\text { Cl16-C5-C4-C3 }=180.000\end{array}$ \\
\hline
\end{tabular}




\begin{tabular}{|c|c|c|c|}
\hline & $\begin{array}{l}\text { Cl15-C4=1.744 } \\
\text { Cl16-C5=1.747 } \\
\mathrm{H} 17-\mathrm{C} 6=1.083 \\
\mathrm{Cl} 18-\mathrm{C} 9=1.733 \\
\mathrm{Cl} 19-\mathrm{C} 10=1.743 \\
\mathrm{H} 20-\mathrm{C} 11=1.083 \\
\mathrm{Cl} 21-\mathrm{C} 12=1.749\end{array}$ & $\begin{array}{l}\text { Cl16-C5-C4=120.858 } \\
\text { H17-C6-C5=119.705 } \\
\text { Cl18-C9-C8=120.184 } \\
\text { Cl19-C10-C9=120.505 } \\
\text { H20-C11-C10=119.299 } \\
\text { Cl21-H20-C11=119.393 }\end{array}$ & $\begin{array}{l}\mathrm{H} 17-\mathrm{C} 6-\mathrm{C} 5-\mathrm{C} 4=180.000 \\
\mathrm{Cl} 18-\mathrm{C} 9-\mathrm{C} 8-\mathrm{C} 7=180.000 \\
\mathrm{Cl} 19-\mathrm{C} 10-\mathrm{C} 9-\mathrm{C} 8=180.000 \\
\mathrm{H} 20-\mathrm{C} 11-\mathrm{C} 10-\mathrm{C} 9=180.000 \\
\mathrm{Cl} 21-\mathrm{H} 20-\mathrm{C} 11-\mathrm{C} 10=180.000\end{array}$ \\
\hline 29 & $\begin{array}{l}\mathrm{C} 2-\mathrm{C} 1=1.410 \\
\mathrm{C} 3-\mathrm{C} 2=1.385 \\
\mathrm{C} 4-\mathrm{C} 3=1.395 \\
\mathrm{C} 5-\mathrm{C} 4=1.402 \\
\mathrm{C} 6-\mathrm{C} 5=1.392 \\
\mathrm{C} 7-\mathrm{C} 1=1.450 \\
\mathrm{C} 8-\mathrm{C} 7=1.405 \\
\mathrm{C} 9-\mathrm{C} 8=1.389 \\
\mathrm{C} 10-\mathrm{C} 9=1.405 \\
\mathrm{C} 11-\mathrm{C} 10=1.414 \\
\mathrm{C} 12-\mathrm{C} 11=1.392 \\
\mathrm{O} 13-\mathrm{C} 8=1.369 \\
\mathrm{H} 14-\mathrm{C} 3=1.083 \\
\mathrm{Cl} 15-\mathrm{C} 4=1.752 \\
\mathrm{H} 16-\mathrm{C} 5=1.083 \\
\mathrm{Cl} 17-\mathrm{C} 6=1.750 \\
\mathrm{Cl} 18-\mathrm{C} 9=1.734 \\
\mathrm{Cl} 19-\mathrm{C} 10=1.735 \\
\mathrm{Cl} 20-\mathrm{C} 11=1.747 \\
\mathrm{H} 21-\mathrm{C} 12=1.082\end{array}$ & $\begin{array}{l}\text { C3-C2-C1=124.421 } \\
\text { C4-C3-C2=115.657 } \\
\text { C5-C4-C3=122.619 } \\
\text { C6-C5-C4=119.538 } \\
\text { C7-C1-C6=136.910 } \\
\text { C8-C7-C1=104.892 } \\
\text { C9-C8-C7=123.028 } \\
\text { C10-C9-C8=117.495 } \\
\text { C11-C10-C9=119.797 } \\
\text { C12-C11-C10=121.826 } \\
\text { O13-C8-C7 =112.186 } \\
\text { H14-C3-C2=122.097 } \\
\text { Cl15-C4-C3=118.898 } \\
\text { H16-C5-C4=120.278 } \\
\text { Cl17-C6-C5=119.254 } \\
\text { Cl18-C9-C8=119.624 } \\
\text { Cl19-C10-C9=119.587 } \\
\text { Cl20-C11-C10=120.327 } \\
\text { H21-Cl20-C11=119.854 }\end{array}$ & $\begin{array}{l}\text { C4-C3-C2-C1=0.000 } \\
\text { C5-C4-C3-C2 =0.000 } \\
\text { C6-C5-C4-C3=0.000 } \\
\text { C7-C1-C6-C5=180.000 } \\
\text { C8-C7-C1-C6=180.000 } \\
\text { C9-C8-C7-C1=180.000 } \\
\text { C10-C9-C8-C7=0.000 } \\
\text { C11-C10-C9-C8=0.000 } \\
\text { C12-C11-C10-C9=0.000 } \\
\text { O13-C8-C7-C1 =0.000 } \\
\text { H14-C3-C2-C1=180.000 } \\
\text { Cl15-C4-C3-C2=180.000 } \\
\text { H16-C5-C4-C3=180.000 } \\
\text { Cl17-C6-C5-C4=180.000 } \\
\text { Cl18-C9-C8-C7=180.000 } \\
\text { Cl19-C10-C9-C8=180.000 } \\
\text { Cl20-C11-C10-C9=180.000 } \\
\text { H21-Cl20-C11-C10=180.000 }\end{array}$ \\
\hline 30 & $\begin{array}{l}\mathrm{C} 2-\mathrm{C} 1=1.407 \\
\mathrm{C} 3-\mathrm{C} 2=1.384 \\
\mathrm{C} 4-\mathrm{C} 3=1.396 \\
\mathrm{C} 5-\mathrm{C} 4=1.412 \\
\mathrm{C} 6-\mathrm{C} 5=1.394 \\
\mathrm{C} 7-\mathrm{C} 1=1.451 \\
\mathrm{C} 8-\mathrm{C} 7=1.407 \\
\mathrm{C} 9-\mathrm{C} 8=1.383 \\
\mathrm{C} 10-\mathrm{C} 9=1.394 \\
\mathrm{C} 11-\mathrm{C} 10=1.414 \\
\mathrm{C} 12-\mathrm{C} 11=1.399 \\
\mathrm{O} 13-\mathrm{C} 8=1.371 \\
\mathrm{H} 14-\mathrm{C} 3=1.083 \\
\mathrm{Cl} 15-\mathrm{C} 4=1.744 \\
\mathrm{Cl} 16-\mathrm{C} 5=1.747 \\
\mathrm{H} 17-\mathrm{C} 6=1.082 \\
\mathrm{H} 18-\mathrm{C} 9=1.083 \\
\mathrm{Cl} 19-\mathrm{C} 10=1.744 \\
\mathrm{Cl} 20-\mathrm{C} 11=1.738 \\
\mathrm{Cl} 21-\mathrm{C} 12=1.742\end{array}$ & $\begin{array}{l}\text { C3-C2-C1=123.507 } \\
\text { C4-C3-C2=116.983 } \\
\text { C5-C4-C3=120.870 } \\
\text { C6-C5-C4=120.975 } \\
\text { C7-C1-C6=136.121 } \\
\text { C8-C7-C1=105.437 } \\
\text { C9-C8-C7=123.798 } \\
\text { C10-C9-C8=116.804 } \\
\text { C11-C10-C9=121.778 } \\
\text { C12-C11-C10=136.242 } \\
\text { O13-C8-C7 =111.720 } \\
\text { H14-C3-C2=121.885 } \\
\text { Cl15-C4-C3=118.002 } \\
\text { Cl16-C5-C4=120.844 } \\
\text { H17-C6-C5=119.667 } \\
\text { H18-C9-C8=121.972 } \\
\text { Cl19-C10-C9=117.637 } \\
\text { Cl20-C11-C10=120.253 } \\
\text { Cl21-Cl20-C11=119.286 }\end{array}$ & 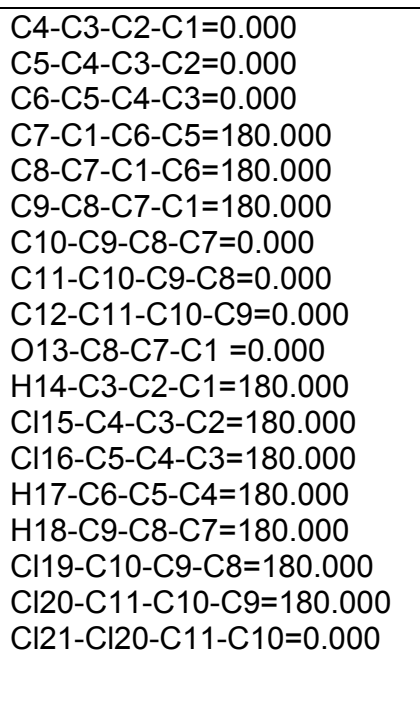 \\
\hline $31=64$ & $\begin{array}{l}\mathrm{C} 2-\mathrm{C} 1=1.408 \\
\mathrm{C} 3-\mathrm{C} 2=1.390 \\
\mathrm{C} 4-\mathrm{C} 3=1.393 \\
\mathrm{C} 5-\mathrm{C} 4=1.403 \\
\mathrm{C} 6-\mathrm{C} 5=1.400 \\
\mathrm{C} 7-\mathrm{C} 1=1.454 \\
\mathrm{C} 8-\mathrm{C} 7=1.408 \\
\mathrm{C} 9-\mathrm{C} 8=1.390 \\
\mathrm{C} 10-\mathrm{C} 9=1.391 \\
\mathrm{C} 11-\mathrm{C} 10=1.404 \\
\mathrm{C} 12-\mathrm{C} 11=1.400 \\
\mathrm{O} 13-\mathrm{C} 8=1.370 \\
\mathrm{Cl} 14-\mathrm{C} 3=1.742 \\
\mathrm{H} 15-\mathrm{C} 4=1.083 \\
\mathrm{Cl} 16-\mathrm{C} 5=1.756 \\
\mathrm{H} 17-\mathrm{C} 6=1.082 \\
\mathrm{Cl} 18-\mathrm{C} 9=1.740 \\
\mathrm{H} 19-\mathrm{C} 10=1.083 \\
\end{array}$ & $\begin{array}{l}\text { C3-C2-C1=122.298 } \\
\text { C4-C3-C2=117.874 } \\
\text { C5-C4-C3=119.856 } \\
\text { C6-C5-C4=119.903 } \\
\text { C7-C1-C6=135.318 } \\
\text { C8-C7-C1=105.180 } \\
\text { C9-C8-C7=122.611 } \\
\text { C10-C9-C8=117.603 } \\
\text { C11-C10-C9=120.892 } \\
\text { C12-C11-C10=121.217 } \\
\text { O13-C8-C7 =112.190 } \\
\text { Cl14-C3-C2=120.977 } \\
\mathrm{H} 15-C 4-C 3=119.864 \\
\text { Cl16-C5-C4=118.259 } \\
\mathrm{H} 17-C 6-C 5=121.781 \\
\text { Cl18-C9-C8=121.074 } \\
\text { H19-C10-C9=119.774 } \\
\text { Cl20-C11-C10=117.760 }\end{array}$ & $\begin{array}{l}\text { C4-C3-C2-C1=0.000 } \\
\text { C5-C4-C3-C2=0.000 } \\
\text { C6-C5-C4-C3=0.000 } \\
\text { C7-C1-C6-C5=180.000 } \\
\text { C8-C7-C1-C6=180.000 } \\
\text { C9-C8-C7-C1=180.000 } \\
\text { C10-C9-C8-C7=0.000 } \\
\text { C11-C10-C9-C8=0.000 } \\
\text { C12-C11-C10-C9=0.000 } \\
\text { O13-C8-C7-C1 =180.000 } \\
\text { Cl14-C3-C2-C1=180.000 } \\
\text { H15-C4-C3-C2=180.000 } \\
\text { Cl16-C5-C4-C3=180.000 } \\
\text { H17-C6-C5-C4=180.000 } \\
\text { Cl18-C9-C8-C7=180.000 } \\
\text { Cl19-C10-C9-C8=180.000 } \\
\text { Cl20-C11-C10-C9=180.000 } \\
\text { Cl21-Cl20-C11-C10=180.000 }\end{array}$ \\
\hline
\end{tabular}


$\mathrm{Cl} 20-\mathrm{C} 11=1.746$

$\mathrm{Cl} 21-\mathrm{C} 12=1.741$

$\mathrm{Cl} 21-\mathrm{Cl} 20-\mathrm{C} 11=121.553$

Table 2: Energy (au), Hardness (eV), Chemical Potential (eV), Electrophilicity (eV) and Local Electrophilicities (eV) (MPA and HPA) for Different Polychlorinated Dibenzofurans.

\begin{tabular}{|c|c|c|c|c|c|c|}
\hline Molecule & $\begin{array}{l}\text { Energy } \\
\text { (a.u.) }\end{array}$ & $\begin{array}{c}\eta \\
(\mathrm{eV})\end{array}$ & $\begin{array}{c}\mu \\
(\mathrm{eV})\end{array}$ & $\begin{array}{c}\omega \\
(\mathrm{eV})\end{array}$ & $\begin{array}{l}\omega_{\text {Max }}^{+} \\
(\mathrm{eV}) \quad \mathrm{MPA}\end{array}$ & $\begin{array}{l}\omega_{\text {Max }}^{+} \\
(\mathrm{eV}) \quad \mathrm{HPA}\end{array}$ \\
\hline 1 & -996.924 & 2.493 & -3.712 & 2.763 & 0.429 & 0.224 \\
\hline 2 & -996.922 & 2.528 & -3.694 & 2.699 & 0.430 & 0.251 \\
\hline 3 & -1456.516 & 2.452 & -3.999 & 3.101 & 0.466 & 0.273 \\
\hline 4 & -1456.518 & 2.453 & -3.950 & 3.180 & 0.466 & 0.248 \\
\hline 5 & -1916.110 & 2.451 & -4.062 & 3.366 & 0.511 & 0.343 \\
\hline 6 & -1916.112 & 2.399 & -4.054 & 3.425 & 0.512 & 0.346 \\
\hline 7 & -1916.100 & 2.439 & -4.031 & 3.331 & 0.530 & 0.326 \\
\hline 8 & -1916.108 & 2.414 & -4.103 & 3.486 & 0.538 & 0.345 \\
\hline 9 & -2375.691 & 2.412 & -4.201 & 3.659 & 0.565 & 0.351 \\
\hline 10 & -2375.694 & 2.392 & -4.234 & 3.748 & 0.570 & 0.360 \\
\hline 11 & -2375.702 & 2.400 & -4.253 & 3.769 & 0.569 & 0.373 \\
\hline 12 & -2375.697 & 2.347 & -4.215 & 3.785 & 0.548 & 0.360 \\
\hline 13 & -2375.698 & 2.363 & -4.202 & 3.736 & 0.599 & 0.329 \\
\hline 14 & -2835.285 & 2.403 & -4.358 & 3.952 & 0.611 & 0.375 \\
\hline 15 & -2835.282 & 2.340 & -4.309 & 3.967 & 0.617 & 0.377 \\
\hline 16 & -2835.285 & 2.329 & -4.319 & 4.005 & 0.579 & 0.376 \\
\hline 17 & -2835.283 & 2.343 & -4.354 & 4.046 & 0.586 & 0.376 \\
\hline 18 & -3294.871 & 2.309 & -4.437 & 4.263 & 0.638 & 0.388 \\
\hline 19 & -3294.868 & 2.342 & -4.485 & 4.293 & 0.603 & 0.386 \\
\hline 20 & -2375.698 & 2.402 & -4.288 & 3.828 & 0.566 & 0.367 \\
\hline 21 & -2375.694 & 2.414 & -4.193 & 3.642 & 0.571 & 0.357 \\
\hline 22 & -2375.697 & 2.356 & -4.151 & 3.657 & 0.559 & 0.351 \\
\hline 23 & -2835.288 & 2.325 & -4.307 & 3.989 & 0.586 & 0.379 \\
\hline 24 & -2835.287 & 2.349 & -4.328 & 3.988 & 0.591 & 0.379 \\
\hline 25 & -2835.278 & 2.330 & -4.323 & 4.010 & 0.579 & 0.377 \\
\hline 26 & -2375.694 & 2.366 & -4.179 & 3.691 & 0.556 & 0.354 \\
\hline 27 & -2375.697 & 2.356 & -4.151 & 3.657 & 0.558 & 0.351 \\
\hline 28 & -2835.288 & 2.325 & -4.307 & 3.989 & 0.586 & 0.379 \\
\hline 29 & -2835.287 & 2.349 & -4.328 & 3.988 & 0.591 & 0.379 \\
\hline 30 & -2835.285 & 2.329 & -4.319 & 4.005 & 0.579 & 0.376 \\
\hline 31 & -2835.288 & 2.338 & -4.361 & 4.067 & 0.633 & 0.342 \\
\hline
\end{tabular}

Table 3: Hardness (eV), Chemical Potential (eV), Electrophilicity (eV) of Nucleic Acid Bases/DNA Base Pairs.

\begin{tabular}{|l|c|c|c|}
\hline Moleule & $\begin{array}{c}\eta \\
(\mathrm{eV})\end{array}$ & $\begin{array}{c}\mu \\
(\mathrm{eV})\end{array}$ & $\begin{array}{c}\omega \\
\mathrm{eV}\end{array}$ \\
\hline Adenine & 2.850 & -3.103 & 1.689 \\
\hline Thymine & 2.894 & -3.689 & 2.351 \\
\hline Guanine & 2.916 & -2.648 & 1.202 \\
\hline Cytosine & 2.785 & -3.370 & 2.039 \\
\hline Uracil & 2.962 & -3.919 & 2.593 \\
\hline GCWC & 2.018 & -3.030 & 2.275 \\
\hline ATH & 2.526 & -3.256 & 2.099 \\
\hline
\end{tabular}

